\title{
The 11th International Conference on Continuous Renal Replacement Therapies (CRRT)
}

March 2-4, 2006, San Diego, Calif.

\section{Abstracts}

Editor

R.L. Mehta, San Diego, Calif.

\section{Contents}

Patient Characteristics

Abstracts 1-11

Emerging Concepts in ARF and CRRT

Abstracts 12-23

Technique Characteristics

Abstracts 24-42

CRRT Research

Abstracts $43-52$

Author Index 


\section{Patient Characteristics}

\section{1} Evaluation of the PRISMA M10 Circuit in
Critically III Infants Requiring Continuous
Renal Replacement Therapy (CRRT):
A Report from the ppCRRT Registry Group S.L. Goldstein', P.D. Brophy², T.E. Bunchman³, R. Hackbarth ${ }^{3}$, D. Blowey

${ }^{1}$ Baylor College of Medicine, Houston, Texas, ${ }^{2}$ University of Michigan, Ann Arbor, 'DeVos Children's Hospital, Grand Rapids, Michigan, ${ }^{4}$ Children's Mercy Hospital and Clinics, Kasnas City, Missouri, USA

Currently available extracorporeal circuits in the US often require blood priming to prevent hypotension/anemia in smaller pediatric patients. The PRISMA M10 circuit, composed on an AN-69 membrane with a blood volume of $50 \mathrm{ml}$ is available in other countries, but has not been cleared by the FDA for use in the US. An IDE study of the M10 circuit was conducted in the US for use in critically ill pediatric patients with acute kidney injury $<15 \mathrm{~kg}$ in size. The IDE study protocol allowed for maximal blood pump flow of $20 \mathrm{ml} / \mathrm{min}$. Fifteen patients $(9 \mathrm{M}, 6 \mathrm{~F}$, mean size $5.8+2.8 \mathrm{~kg}$, range $2.6-12.5 \mathrm{~kg}$, age $4 \mathrm{~d}-13 \mathrm{mo}$, mean creatinine $=1.2+0.7 \mathrm{mg} / \mathrm{dl}$ ) were studied at 4 Prospective Pediatric CRRT Registry Group centers. A total of 61 filters (range 1-4 circuits per pt) were used (mean circuit life $28.6+22.5 \mathrm{hrs}$, range 1 to $74.5 \mathrm{hrs}, 55 \%>24 \mathrm{hrs}$ ) and no blood leaks occurred. All circuits achieved Qb $20 \mathrm{ml} / \mathrm{min}$. 42/61 filters clotted and mean circuit life was lower for these filters than those changed for other reasons $(23+17$ vs. $41+28 \mathrm{hrs}, \mathrm{p}<0.005)$. Mean total UF was $7893+12962 \mathrm{ml}(-148$ to $40283 \mathrm{ml})$ with $12 / 15$ patients surviving and $3 / 15$ patients achieving dry weight. We conclude that the PRISMA M10 circuit can serve well for CRRT in small pediatric patients. Further research is needed to determine if higher blood pump flow rates would decrease clotting incidence and increase filter life span and ultrafiltration rates.
2 Metabolic Acidosis in Patients with Liver
Failure Undergoing Regional Citrate
Anticoagulation for CVVHDF

J. Bouchard, M. Vallée, F. Madore

Nephrology Division, Hôpital du Sacré-Coeur de Montréal, Canada

Background: Citrate anticoagulation is increasingly used for CRRT. Recently, it has been suggested that its use can be safe in patients with liver failure. However, significant complications, such as metabolic acidosis due to impaired citrate metabolism, can occur. Methods: We reviewed the utilization of citrate anticoagulation for CRRT in patients with hepatic injury in our center. For all patients, sodium citrate $4 \%$ was initiated at $220 \mathrm{ml} / \mathrm{hr}$ and adjusted to maintain postfilter ionized calcium between $0.25-0.35 \mathrm{mmol} / \mathrm{L}(1.0-1.4 \mathrm{mg} / \mathrm{dl})$. Calcium chloride $1.5 \%$ was initiated at $20 \mathrm{ml} / \mathrm{hr}$ and adjusted to maintain systemic serum calcium between $1.0-1.3 \mathrm{mmol} / \mathrm{L}(4.0-5.3 \mathrm{mg} / \mathrm{dl})$. Blood flow rate was $125 \mathrm{ml} / \mathrm{hr}$. Sodium chloride $0.9 \%$ was infused at $1000 \mathrm{ml} / \mathrm{hr}$ as replacement fluid. The dialysate, composed of $4750 \mathrm{ml}$ of Hemosol B0 solution (Gambro), was delivered at $1000 \mathrm{ml} / \mathrm{hr}$ after having discarded the $250 \mathrm{ml}$ of $\mathrm{NaHCO}_{3}$ solution. Results: Between January 2004 and September 2005, 15 patients underwent citrate anticoagulation for CVVHDF treatments, due to filters coagulation problems. Three patients presented with liver injury (acute ischemic liver injury, metastatic hepatocarcinoma with peripheral signs of cirrhosis, and chronic Child $\mathrm{C}$ cirrhosis). No modification was required to our citrate protocol and no complications occurred in $2 / 3$ of cases. One patient (Child C cirrhosis) developed in $<8$ hours severe metabolic acidosis associated with increased total to ionized calcium ratio $(>2.5)$, suggesting an accumulation of unmetabolized citrate. Bicarbonate levels decreased from 21 to $14 \mathrm{mEq} / \mathrm{L}$. A bicarbonate perfusion and a reduction in the citrate perfusion rate did not correct the acidosis. The dialysate composition was modified to add the bicarbonate solution $(250 \mathrm{ml})$ to the Hemosol B0 $(4750 \mathrm{ml})$ solution and the dialysate infusion rate was increased to $2000 \mathrm{ml} / \mathrm{hr}$. Bicarbonate levels returned to normal values and remained stable. Conclusion: Citrate anticoagulation may be used in patients with hepatic injury, but important precautions must be taken, especially in cirrhotic patients. Acid-base status and total to ionized calcium ratio should be closely monitored with concerns about metabolic acidosis and accumulation of unmetabolized citrate. In such cases, adjustments in the rate of bicarbonate, citrate and possibly calcium perfusions should be undertaken.

\begin{tabular}{ll}
\hline KARGER & (c) 2006 S. Karger AG, Basel \\
Fax +4161306 1234 & 0253-5068/06/0243-0248\$23.50/0 \\
$\begin{array}{l}\text { E-Mail karger@karger.ch } \\
\text { www.karger.com }\end{array}$ & $\begin{array}{l}\text { Accessible online at: } \\
\text { www.karger.com/journals/bpu }\end{array}$
\end{tabular}


3

\section{CRRT in Developing Countries}

V. Avadaiammal, M. Leelakumari, J. George, B. Upendran, S. Varma, M.K. Mohandas

Medical College Hospital, Trivandrum, India

This study is a retrospective analysis of aetiology and outcome of CRRT in patients with renal dysfunction and hemodynamic instability in a tertiary care centre in Kerala, India. All patients admitted to Medical College Hospital, Trivandrum requiring CRRT between the period January 1, 2003 to December 31, 2004 were included in the study. All patients in whom CRRT was discontinued within 6 hours due to any cause was excluded from the study. 66 patients were studied with a mean age of $41.74 \pm 15.32$ years. Male to female ratio was $1.75: 1$. Patients predominantly had acute renal failure (88\%). $6 \%$ each had acute on chronic and chronic renal failure. $63.6 \%$ of patients survived. In spite of limitations due to financial constraints, (PD fluid was used as the dialysate, F8 dialyzer was used in many instances instead of $\mathrm{CVVH}$ filter, lack of adequate facilities for frequent biochemical monitoring), CVVHD remains an important modality of renal support in patients with renal dysfunction in developing countries.

\section{4 \\ RIFLE Classification Does Not Predict Outcome in Dialytic Acute Kidney Dysfunction}

\author{
E. Maccariello', C. Valente', L. Nogueira', M. Soares², \\ E. Rocha' \\ ${ }^{1}$ NepHro; Rede D'Or de Hospitais; Universidade Federal \\ do Rio de Janeiro, ${ }^{2} \mathrm{NepHro}$; Instituto Nacional de Cancer; \\ Rio de Janeiro, RJ, Brazil
}

Purpose: To prospectively determine the characteristics and prognosis of patients with Acute Kidney Dysfunction (AKD) who required dialysis support at the Intensive Care Unit (ICU) and to validate the RIFLE classification in this population. Methods: Over one year, 214 patients that were admitted to the ICU at three tertiary care hospitals and required renal replacement therapy (RRT) were studied. Patients with end-stage renal disease requiring chronic dialysis and those with ICU stay less than $24 \mathrm{~h}$ were excluded. AKD was classified according to RIFLE criteria. Data were collected at the start of RRT. Logistic regression was used in multivariable analysis and results are presented as odds-ratio and 95\% confidence interval (OR, 95\% CI). Results: The mean age was 71.4 years [standard deviation $(\mathrm{SD})=15.8$ ]; 112 $(52 \%)$ patients were males. The SAPS II and SOFA (except renal domain) scores were $47.9(\mathrm{SD}=10.8)$ and $6.0(\mathrm{SD}=3.1)$ points, respectively; 177 (83\%) were on mechanical ventilation and $157(73 \%)$ used vasopressors. The main reasons for AKD were sepsis (74\%), ischemia/shock (72\%) and drug/toxins (29\%). Continuous RRT were initially used in $179(84 \%)$ patients and the main indications for RRT were acidosis $(72 \%)$, hypervolemia $(50 \%)$, uremia $(43 \%)$ and anuria (37\%). According to RIFLE criteria, patients were classified as risk $(\mathrm{n}=54,25 \%)$, injury $(\mathrm{n}=58,27 \%)$ and failure $(\mathrm{n}=102,48 \%)$. The overall mortality rate was $76 \%$ and there were no differences according

The 11th International Conference on

Continuous Renal Replacement

Therapies (CRRT) to the RIFLE classification (risk $=72 \%$, injury $=79 \%$ and failure $=76 \%, p=0.681)$. The final model of multivariable analysis was: age (years) $[\mathrm{OR}=1.04(1.01-1.31), \mathrm{p}=0.004]$, need of vasopressors $[\mathrm{OR}=2.72(0.99-7.49), \quad \mathrm{p}=0.053 ;$ presence of comorbidity (Charlson index $>1$ ) $[\mathrm{OR}=2.75(1.01-7.51), \mathrm{p}=0.048]$, impaired functional capacity $[\mathrm{OR}=7.69(2.33-25.42), \mathrm{p}=0.001]$ and more than two associated organ failures $[\mathrm{OR}=10.66$ (2.13-53.35), $\mathrm{p}<0.001]$. The RIFLE classification was forced into the model and was not selected. Conclusions: Age, need of vasopressors, more than two associated organ failures, the presence of comorbidity and a previous reduced functional capacity were significantly related to a higher mortality in this population. The RIFLE classification did not discriminate the prognosis in the ARF patients who underwent dialysis support in the ICU.

5

Therapy induced Alkalosis Is Not Associated
with Increased Mortality in Intensive Care
Unit (ICU) Patients on Continuous Renal
Replacement Therapy (CRRT)

S.G. Demirjian, B.W. Teo, K. Howey, E.P. Paganini

Cleveland Clinic Foundation, USA

Background: Acid-base disorders are common in critically ill patients. Once CRRT is initiated, it becomes a major determinant of acid-base status. We hypothesized that therapy induced alkalosis is associated with increased hospital mortality. Methods: Prospective observational cohort of ICU patients requiring CRRT at the Cleveland Clinic between 1995 and 2001. 274 patients who were on bicarbonate based CRRT for 3 days or more, and had arterial $\mathrm{pH}$ measurements on day four were included in the study. Acid-base status was analyzed after 3,5 , and 10 days of CRRT (acidosis $\mathrm{pH}<7.35$, alkalosis $\mathrm{pH}>7.45$ ). Univariate, followed by multivariate linear regression analyses were performed when appropriate. Mixed modeling (proc mixed) was used in analyzing repeated measures. All statistical analyses were performed with SAS v8. Results: Baseline characteristics: Age $59.7 \pm 15 \mathrm{yrs}$, $64 \%$ male, dose of dialysis $15.6 \pm 6.5 \mathrm{~mL} / \mathrm{kg} / \mathrm{hr}, \mathrm{pH} 7.38 \pm 0.1, \mathrm{CO}_{2}$ $21.2 \pm 4.5 \mathrm{mmol} / \mathrm{L}, \mathrm{PaCO}_{2} 37.6 \pm 10.8 \mathrm{mmHg}$. In univariate analysis, at day 3,5 , and 10 , alkalosis was not associated with increased mortality compared to normal $\mathrm{pH}(39.2$ vs. $41.7 \%, 37.0$ vs. $36.5 \%, 34.3$ vs. $39.3 \%)$. But acidosis was associated with higher mortality at day 3 and $5(65.4$ vs. $41.7 \%, p=0.004,60$ vs. $36.5 \%, p=0.04)$. Independent predictors of $\mathrm{pH}$ are shown in table 1 . Non-survivors had lower $\mathrm{pH}$ initially and throughout CRRT after adjusting to potential confounders using mixed modeling, see figure 1. Conclusion: Alkalosis during CRRT is not associated with increased mortality. Acidosis at initiation and during CRRT was associated with increased mortality.

Blood Purif 2006;24:247-273 249 
Table 1. Independent predictors of $\mathrm{pH}$ at days 3, 5 and 10 by multiple linear regression

\begin{tabular}{|c|c|c|c|c|c|c|}
\hline \multirow[t]{2}{*}{ Variables* } & \multicolumn{2}{|l|}{ Day 3} & \multicolumn{2}{|l|}{ Day 5} & \multicolumn{2}{|l|}{ Day 10} \\
\hline & $\begin{array}{l}\text { Parameter } \\
\text { estimate }\end{array}$ & $P$ value & $\begin{array}{l}\text { Parameter } \\
\text { estimate }\end{array}$ & P value & $\begin{array}{l}\text { Parameter } \\
\text { estimate }\end{array}$ & P value \\
\hline $\mathrm{CO}_{2}$ & 0.013 & $<0.0001$ & 0.015 & $<0.0001$ & 0.012 & $<0.0001$ \\
\hline $\begin{array}{l}\text { Anion gap } \\
\mathrm{PaCO}_{2}\end{array}$ & -0.008 & & $\begin{array}{r}0.004 \\
-0.008\end{array}$ & $\begin{array}{l}<0.0001 \\
<0.0001\end{array}$ & -0.008 & \\
\hline $\begin{array}{l}\text { Respiratory } \\
\text { failure }\end{array}$ & & & 0.023 & 0.008 & & \\
\hline Dialysis dose & 0.00004 & 0.03 & 0.00005 & 0.01 & & \\
\hline WBC count & & & -0.001 & 0.004 & & \\
\hline $\begin{array}{l}\text { Adjusted } \\
\mathrm{R}_{2}\end{array}$ & 0.75 & & 0.76 & & 0.70 & \\
\hline
\end{tabular}

* Variables included in the preliminary multiple linear regression model were: age, gender, $\mathrm{PaCO}_{2}$, mean arterial pressure, dialysis dose, WBC count, anion gap, BUN, and mechanical ventilation.

Figure 1. Non-survivors had lower $\mathrm{pH}$ values at initiation that persisted throughout CRRT.

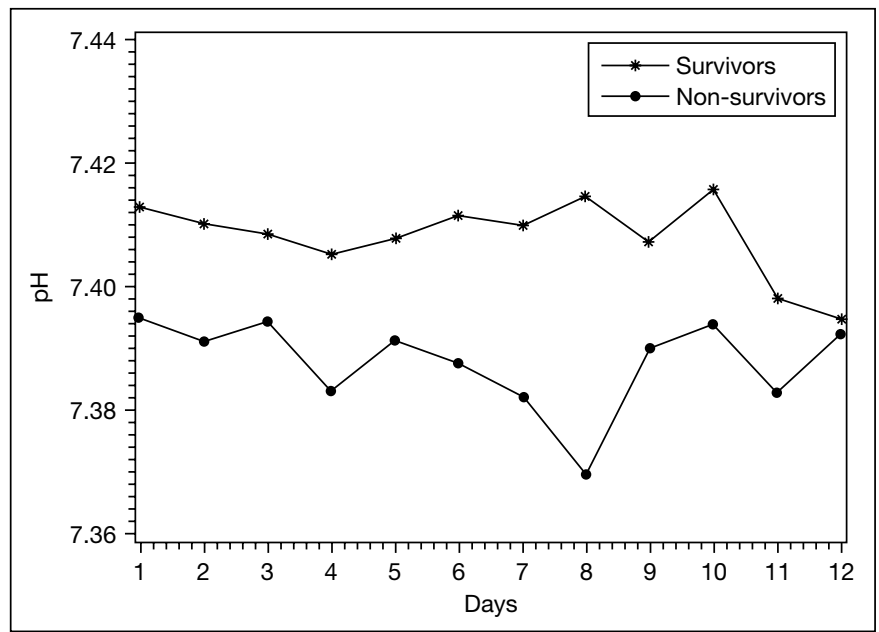

*Adjusted for age, gender, dialysis dose, $\mathrm{CO}_{2}, \mathrm{PaCO}_{2}, \mathrm{BUN}$, hematocrit, aniongap, $\log$ urine output, mean arterial pressure, pressor requirements, and mechanical ventilation.
6 CRRT in Pediatric Sepsis: A Report from the
Prospective Pediatric Continuous Renal
Replacement Therapy (ppCRRT) Registry
Group

F.X. Flores, P.D. Brophy, J.M. Symons, J. Fortenberry,

A.N. Chua, S.R. Alexander, J. Mahan, T.E. Bunchman,

D. Blowey, M.J.G. Somers, M. Baum, R. Hackbarth,

D. Chand, K. McBryde, S.L. Goldstein

The ppCRRT Registry Group, Houston, Texas, USA

CRRT has become more prevalent in the management of fluid overload (FO) and electrolyte imbalance in pediatric patients with Acute Kidney Injury (AKI). Since 01/01/01 115 of 370 patients enrolled in the 13-center ppCRRT Registry have a primary or secondary diagnosis of sepsis. Complete data for 102 patients with sepsis were available for analysis. Median age was 7.32 years (range 2 days -25.11 years), 44 $(43 \%)$ were female, $61(60 \%)$ received diuretic therapy, $81(79 \%)$ had MODS and $83(81 \%)$ required ventilatory support. CRRT modalities were CVVHD (52\%), CVVH (26\%) and CVVHDF (22\%). Indications to initiate CRRT were fluid and electrolyte abnormalities (54\%), FO only (38\%) and electrolyte abnormality only (8\%). 57 (56\%) patients survived (S) and ventilated patients had similar $\mathrm{S}$ as non-ventilated patients $(53 \%$ vs. $68 \%, \mathrm{p}=0.22)$. There was no significant difference in the $\mathrm{S}$ rate among CRRT modalities [CVVHD (47\%) vs. CVVH (67\%) vs. CVVHDF (64\%), $p=0.19]$. Survivors had lower FO at CRRT initiation and Paw at CRRT termination.

\begin{tabular}{lccr}
\hline & \multicolumn{1}{c}{ Survivors (S) } & Non-survivors (NS) & P value \\
\hline Age (yrs) & $9.01 \pm 0.93$ & $8.07 \pm 1.04$ & 0.50 \\
Initial PRISM 2 & $14.08 \pm 1.25$ & $17.00 \pm 1.36$ & 0.07 \\
Initial Paw & $19.27 \pm 1.59$ & $20.89 \pm 1.69$ & 0.40 \\
FO at CRRT (\%) & $15.45 \pm 3.85$ & $28.13 \pm 4.33$ & $<0.05$ \\
GFR & $30.43 \pm 3.25$ & $29.82 \pm 3.17$ & 0.96 \\
CVP (cm H $\left.H_{2} \mathrm{O}\right)$ & $16.37 \pm 1.26$ & $17.87 \pm 1.32$ & 0.41 \\
Number of pressors & $1.58 \pm 0.15$ & $1.88 \pm 0.17$ & 0.19 \\
Time ICU to & $4.73 \pm 2.46$ & $10.60 \pm 2.70$ & 0.11 \\
$\quad$ CRRT (days) & & & \\
CRRT duration & $9.50 \pm 5.30$ & $19.25 \pm 6.03$ & 0.26 \\
$\quad($ days) & & & \\
UF volume (ml) & $50570.87 \pm 6931.30$ & $44749.8 \pm 7800.92$ & 0.57 \\
Clearance & $2217.01 \pm 60.50$ & $2650.56 \pm 174.90$ & $<0.05$ \\
$\quad\left(\mathrm{ml} / \mathrm{hr} / 1.73 \mathrm{~m}^{2}\right)$ & & & \\
Paw at end CRRT & $10.84 \pm 1.27$ & $19.72 \pm 1.30$ & $<0.05$ \\
\hline
\end{tabular}

All values are mean $\pm \mathrm{SE}$

These ppCRRT Registry data show: (1) significant difference between $\mathrm{S}$ and NS in FO\% at initiation of CRRT, (2) improvement in S pulmonary status at the end of CRRT and (3) a trend toward improved survival with convective therapy. We suggest (1) early CRRT initiation after resuscitation to balance fluid status may improve both pulmonary function and survival in pediatric patients with sepsis and (2) the currently accepted belief that convective therapy offers therapeutic advantage over diffusion may be justified. 
7

\section{Incidence, Clinical Predictors and Outcome of Acute Renal Failure Among Trauma Patients: A Prospective Multicenter Study}

\author{
A. Bihorac ${ }^{1}$, A.J. Layon'1 , L.L. Moldawer ${ }^{2}$ \\ ${ }^{1}$ Division of Critical Care, Department of Anesthesiology, \\ ${ }^{2}$ Department of Surgery, University of Florida, \\ Gainesville, USA
}

Data on epidemiology, clinical predictors and outcomes of acute renal failure (ARF) among severe trauma patients is limited and mostly retrospective in nature. The incidence of ARF varies between $0.1-8.4 \%$ due to inconsistent definitions of ARF. Several retrospective studies reported high mortality of $40-70 \%$ for trauma patients with severe ARF. We performed a secondary analysis of an existing database of the 'Inflammation and the Host Response to Injury' (Glue Grant) study which prospectively collected clinical data on patients with severe trauma in multiple clinical centers. ARF was defined using recently published Rifle criteria on the basis of the change in the serial serum creatinine values. 727 patients' data were assessed from database. Complete data were available for 561 patients. After review of each individual patient file, we identified 109 (19\%) patients with ARF as defined by Rifle criteria: 35\% with Rifle-Risk (R), 32\% with Rifle-Injury (I) and 33\% with Rifle-Failure (F). Patients who developed ARF were more likely to be older Caucasian males. Significantly lower systolic but not diastolic or mean arterial blood pressures in emergency room (ER), but not on the scene of accident, characterized patients who developed ARF. Serum lactate levels in ER were also significantly higher among patients who later developed ARF. APACHE II score at the time of ICU admission was significantly higher among ARF patients. These patients had prolonged ICU hospital stay, required longer mechanical ventilation and more use of steroids. Hospital mortality (42\%) was significantly worse for patient with ARF, compared to patients with normal renal function $(17 \%)$. Patients with ARF had significantly higher prevalence of both infectious and non-infectious complications. Sepsis and multi-organ failure were more common causes of death among patients with ARF.

Figure 1.

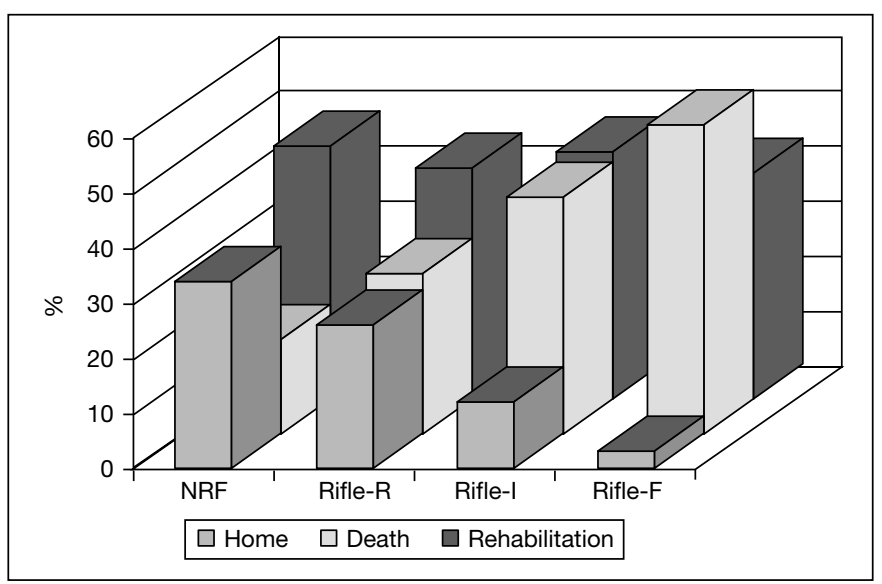

The 11th International Conference on

Continuous Renal Replacement

Therapies (CRRT)
Hospital mortality was correlated to the severity of ARF and was $29 \%, 43 \%$ and $56 \%$ for patients with Rifle-R, Rifle-I and Rifle-F, retrospectively (fig. 1). Incidence of ARF among trauma patients may be higher than previously reported. ARF is a severe complication with significantly increased hospital morbidity and mortality, and severity of ARF correlates with adverse outcomes.

\section{8}

\section{Temporary Vascular Access for Renal Replacement Therapy (RRT) - Predictors of Resource Utilization in an Inner City Population}

R.R.V. Chemitiganti' ${ }^{1}$ P.K. Gona' ${ }^{1}$, P. Krish ${ }^{2}$, M. Difiglia', R.L. Petrillo ${ }^{1}$, Z. Carrey $^{1}$

'The Mount Vernon Hospital, Mount Vernon, NY, USA; ${ }^{2}$ St. Georges University School of Medicine, St. George, Grenada

Temporary Vascular Access (TVA) plays a significant part in delivery of RRT both in patients already on dialysis (with either a transient complication in the usual access or due to absence of an AV access), and also in patients with Acute Renal Failure (ARF). Placement of TVA's, follow-up, associated complications and the high risk of the patients in question requires a significant proportion of the available healthcare resources. Methods: A randomized retrospective chart review $(n=120)$ was used to analyze the length of stay and factors predicting the utilization of resources in an inner city population, admitted to our facility requiring a TVA during years 2003 and 2004. Results: Mean age of the sample was $65.6 \pm 15.8$ years (Males - 60; Females - 60). A majority of TVA (69.7\%) were required for complications in the existing access, $23 \%$ for people with acute deterioration in chronic renal disease and $7.3 \%$ in patients with ARF (no preexisting renal disease). The most common complications in the usual access were thrombus formation (68.2\%), sepsis (24.7\%) and bleeding (4.7\%). Average hospitalization was 11 days after adjusting for outliers. A weekend admission, absence of a primary care physician, ARF, sepsis or bleeding complicating the usual access, significant co-morbidities $(\geq 4)$, Serum Albumin, Age, Gender, Race, Training or background of the physician performing the procedure, Insurance status, Type of insurance did not show any association with the same. Conclusion: Our research highlights the presence of significant predictors for utilization of resources in an inner city population requiring a TVA for RRT. Further research to understand the impact of the same in larger populations and on developing innovative methods to reduce patient morbidity and better manage the ever shrinking health care resources is required. 


\section{9}

\section{Short-time Results Treatment on CRRT in Department of Nephrology in Podgorica}

\author{
M. Ratkovie, V. Vujoševie, H. Lukae, L.J. Pejakov \\ Clinical of Nephrology and Intesive Care, Podgorica, \\ Serbia and Montenegro
}

The patients with ARF (acute renal failure) have been treated by use of one of the CRRT methods for the first time in the Clinical Center Podgorica since 2004. We have provided the apparatus multifiltra made by Fresenius company for hemodialysis of our ARF patients. Our aim with this study is to show and share the results we have achieved in order to improve our further work as well as to acquire new knowledge and to be criticized in goodwill. The data given in this study relate to the period from 01.07.2004-01.11.2005. All our patients were treated with CVVHDF, original kit 8, Fresenius M.care. The number of the patients treated during this period was 13 (11 male patients and 2 female). Average age of male patients was 51.7 and of female patients was 51.5. 12 patients out of 13 treated, developed ARF for the first time while with one female patient renal failure had already been verified and in the noted period got worse. ARF etiology of our patients was as follows:

- 4 patients suffered from Cholecystopancreatitis.

- 4 patients had undergone cardio vascular surgery.

- 2 patients had undergone triple ACBP.

- 2 patients had aneurusmoctomy on abdominal.

- 1 patient had just had cesarean section (8 pregnancy, 3 living babies). ARF was caused by severe hypervalemy and hemorrhagic syndrome. In the further treatment primary antiphospholipid syndrome was diagnosed.

- 1 patient suffered from hemorrhagic pulmonary renal syndrome with previously verified hemorrhagic fever - Hanton virus positive.

- With 1 patient a GN-membranosa gradusii was diagnosed by use of byopsy and the patient developed pulmonary hemorrhagic syndrome as well.

- The last two patients had sepsis which caused the development of ARF. The colostoma infection was preceded by adenocarcinoma colon surgery and incomplete artificial abortion.

- The level of nitrogen substances was: urea $14-39.6 \mathrm{mmol} / \mathrm{L}$; creatinine $280-86 \mathrm{mmol} / \mathrm{L}$.

- The average duration of CVVHDF was 20.65 hours. The longest treatment was on the patient suffered from antiphospolipid syndrome and it lasted 49 hours.

- The average dose of anticoagulation with heparin was $800-1300 \mathrm{IJ} / \mathrm{H}$.

Arterial pump of $200-250 \mathrm{ml} / \mathrm{h}$ the flow of substances for dialysis was $700-1200 \mathrm{ml} / \mathrm{h}$ substituans $1000-1500 \mathrm{ml} / \mathrm{h}$. The value of UF with 12 patients was $450-600 \mathrm{ml} / \mathrm{h}$ and with 1 patient UF was $800-100 \mathrm{ml} / \mathrm{h}$. Total recovery with patients who suffered from primary antphospolid syndrome, while total recovery of renal function was achieved with 6 patients, 2 patients died and the evaluation of the treatment was not possible. The female patient with renal failure previously verified did not show any sign of renal function recovery. The treatment was continued with chronic hemodialysis. The remained 4 patients treated with this method did not show overall improvement neither the ARF was resolved. Conclusion: This was a modest experience; the choice of indications was not quite good, there was no coagulation incidents, necessary replacements of membranes every 8-10 h.
10

\section{Demographic Characteristics of Pediatric CRRT: A Report of the Prospective Pediatric Continuous Renal Replacement Therapy (ppCRRT) Registry Group}

\author{
J.M. Symons, M.J.G. Somers, M.A. Baum, T.E. Bunchman, \\ P.D. Brophy, D. Blowey, J.D. Fortenberry, D. Chand, \\ F.X. Flores, R. Hackbarth, S.R. Alexander, J. Mahan, \\ K.D. McBryde, S.L. Goldstein \\ ppCRRT Registry Group, Houston, Texas, USA
}

Since 1/1/01, 346 patients from 13 US centers have been enrolled in the ppCRRT registry. We now report demographic characteristics and outcomes for 342 patients with complete data. Ages were newborn to 25 years, $59 \%$ males, weights $1.3-160 \mathrm{~kg}$ (mean $33.7 \mathrm{~kg}$, median $24.2 \mathrm{~kg}$ ). Subjects averaged 5.7 days in ICU prior to CRRT (range 0-135, median 2). At CRRT initiation, $48 \%$ were on diuretics and $66 \%$ were on vasoactive drugs. The table lists indications for CRRT and principal diagnoses. 27\% received CVVH alone; $48 \%$ received CVVHD alone; others received multiple modalities. Mean blood flow $\left(\mathrm{Q}_{\mathrm{B}}\right)$ was $97.9 \mathrm{ml} / \mathrm{min}$ (range $10-350 \mathrm{ml} / \mathrm{min}$, median $100 \mathrm{ml} / \mathrm{min}$ ); mean $\mathrm{Q}_{\mathrm{B}}$ per body weight was $5 \mathrm{ml} / \mathrm{min} / \mathrm{kg}$ (range $0.6-53.6 \mathrm{ml} / \mathrm{min} / \mathrm{kg}$, median $4.1 \mathrm{ml} / \mathrm{min} / \mathrm{kg}$ ). Days on CRRT were $<1-83$ (mean 9.1; median 6). Overall survival was $58 \%$. Survival was lowest when CRRT was started for combined fluid overload and electrolyte imbalance. There was better survival in subjects with principal diagnoses of drug intoxication, renal disease, tumor lysis syndrome, and ischemia/shock; survival was lowest in liver disease and bone marrow transplant. Survivors and non-survivors were not significantly different for other reported demographic data. We conclude that CRRT can be used successfully for a wide range of critically ill pediatric patients and that patient survival is best for those with acute, specific abnormalities who lack multiple organ involvement. Earlier use of CRRT may lead to better outcomes; sicker patients with selected diagnoses may have lower survival.

\begin{tabular}{lrrr}
\hline & N & Survivors & \% Survival \\
\hline Indications for CRRT: & & & \\
$\quad$ Fluid overload and electrolyte & 153 & 79 & 52 \\
$\quad$ imbalance & 100 & 61 & 61 \\
$\quad$ Fluid overload only & 44 & 30 & 68 \\
$\quad$ Electrolyte imbalance only & 11 & 7 & 64 \\
Prevent fluid overload to allow intake & 34 & 23 & 68 \\
Other & & & \\
Principal Diagnoses: & 92 & 51 & 55 \\
Sepsis & 55 & 25 & 45 \\
Bone marrow transplant & 37 & 20 & 54 \\
Cardiac disease/transplant & 30 & 9 & 30 \\
Liver disease/transplant & 29 & 15 & 52 \\
Malignancy (no tumor lysis & & & \\
$\quad$ syndrome) & 27 & 25 & 93 \\
$\quad$ Renal disease & 18 & 12 & 67 \\
Inborn error of metabolism & 16 & 13 & 81 \\
Ischemia/shock & 12 & 12 & 100 \\
Drug intoxication & 11 & 9 & 82 \\
Tumor lysis syndrome & 8 & 5 & 63 \\
Pulmonary disease/failure & 7 & 4 & 57 \\
Other & &
\end{tabular}


11

\section{Comparison of Continuous Veno-Venous Hemodialysis with Intermittent Peritoneal Dialysis in ICU Setting}

\author{
Ashwani Gupta, M. Malik, A.K. Bhalla, J. Gaikwad, \\ Anu Gupta, Ankur Gupta, D. Khullar, S. Ojha, P.S. Sastri, \\ B.K. Rao, D.S. Rana \\ GangaRam Hospital, New Delhi, India
}

Intermittent Peritoneal Dialysis (IPD) using a stylocath is an easy, cost effective \& less cumbersome means of renal replacement therapy which can be easily done in Intensive Care Unit (ICU). This study was designed to compare IPD with Continuous Veno-venous Hemodialysis (CVVHD) in ICU settings. A total of 130 patients (65 in CVVHD group, 49 males $\& 16$ females mean age $50.12=17.45$ yrs $\& 65$ patients in IPD group 47 males 18 females mean age $48.24=16.24$ yrs) were retrospectly studied.

In CVVHD group 21 (32.3\%) \& in IPD group 23 (35.4\%) patients survived (statistically NS). In both groups survival correlated with lower APACHE score \& higher MAP at 0,8 , and $24 \mathrm{hrs}$ (P value $<0.001$ in both groups). The URR (urea reduction ratio) was higher in survivors in both groups as compared to non survivors. S/Albumin at baseline was significantly higher in survivors than non survivors in both groups $(2.73$ $=0.81 \mathrm{gm} / \mathrm{dl}$ vs $2.34=0.52$ in CVVHD gr \& $2.80=0.90 \mathrm{gm} / \mathrm{dl}$ vs $2.41=0.62$ in IPD group) Patients in IPD group were sicker with low mean arterial pressure (MAP) requiring inotropic support, $(73.8 \%$ in IPD vs $58.4 \%$ in CVVHD group).

In conclusion IPD offers comparable Survival advantages in critically sick patients with renal failure in ICU settings with low cost.

\section{Emerging Concepts in ARF and CRRT}

12 Implementing a New Technology in a Clinical
Setting: The Ingredients for Success

M.V. Berube

Sacre Coeur Hospital, University of Montreal Teaching Center, Montreal, QC, Canada

Due to ongoing technological development, it is common for critical care units to face change. No matter how many benefits are to be derived from a change, implementing it remains a challenge. The Lewin's model provides a structured approach which facilitates the implementation of a change. This model has been use in our hospital to introduce the Prismaflex. The first phase of Lewin's model consists of analyzing the situation and brainstorming on the plan of actions. During this first phase, we identified and analyzed the many changes related to nursing practice affected by the transition from the Prisma to the Prismaflex. The information was obtained by meeting with Gambro's specialists and attending a six-hour training session for super users. Afterwards, the Clinical Nurse Specialist in collaboration with the medical and nursing teams revised the current hemofiltration orders and protocols to make them appropriate for the new technology. We completed the first phase of the Prismaflex implementation with a three-hour training session provided to the Prisma certified Nurses. Then comes the implementation phase, in which the technology is introduced in the clinical setting. During this phase, support to the users is crucial. In order to provide this support, two Gambro specialists remained on-site until the users felt comfortable with the Prismaflex and 24/7 phone support was available. Unit resource people would regularly be present at the bedside to help users troubleshoot any arising issues. The last phase of the Lewin's change process consists of restoring the practice into a steady state. Hence, the most frequently encountered issues and their solutions were regularly communicated to the nursing team. Meetings were also held with Gambro specialists on an on-going basis to resolve outstanding issues and to discuss new findings on the hemofiltration therapies. Our experience revealed that to be a success, the implementation of a new technology must be well planned and resource personnel's time opened up to support users effectively.

\section{3 \\ Extracorporeal Blood Purification (ECBP) in Adult Respiratory Distress Syndrome (ARDS)}

\author{
P. Visweswar Reddy \\ Cheif Nephrologist, Bollineni Super Speciality Hospital, \\ Nellore, Andhra Pradesh, India
}

Aim: To determine the beneficial effect of ECBP over respiratory and hemodynamic parameters in ARDS. Material and Methods: 16 patients of ARDS were subjected to CVVH/ CVVHDF. Multi organ failure (MOF), ARDS were defined as per the American-European consensus conference and ACCP/SCCM consensus conference committee guide lines and ARF as base line normal creatinine increasing to $>2.5 \mathrm{mg}$ with in 24 hours. Routine labs and imaging studies including echo were performed. Replacement exchange volume, dialysate flow and blood flow rates respectively were $10-20 \mathrm{ml} / \mathrm{kg} / \mathrm{h}, 1$ liter/hour and $100 \mathrm{ml} / \mathrm{mt}$. Results: There were 12 males. Average age was 50.5 years. The diseases associated - post operative 6 , pneumonia 2, pancreatitis 3 , necrotizing fasciitis 2 , burns 2 , poly trauma with poly transfusion 1 . Underlying sepsis was considered in 14 and ARF in 4. Ventilatory and inotropic support was initiated in 14 . CVVH $(n=12) / \operatorname{CVVHDF}(n=4)$ started with in six hours in 6 and after six hours in remaining after admission into ICU. Average duration of procedure was 42 hours (range 20-64 hours). Inotropic drug dose or number of drugs was reduced after $>24$ hours in $7(50 \%)$, with significant improvement in mean arterial pressure mean arterial blood pressure from $55 \pm$ $3 \mathrm{mmHg}$ to $70 \pm 4 \mathrm{mmHg}$. Respiratory parameters $-\mathrm{O}_{2}$ saturation $(89 \%$ $\pm 1.2 \rightarrow 95.2 \% \pm 4.2)$ PEEP level $\left(8.2 \pm 3.5 \rightarrow 6 \pm 4.2 \mathrm{cmH}_{2} \mathrm{O}\right)$ respiratory rate $(28.4 \pm 4.8 \rightarrow 18.2 \pm 5.1)$, Spo2/Fio2 ratio (117.33 \pm $4.2 \rightarrow 2.11 \pm 2.6)$ improved in $12(75 \%)$. Normalization of chest $\mathrm{X}$ ray was noted in all responders, and in $4(25 \%)$ non-responders. Five patients -4 patients in whom procedure initiated with in 6 hours in 1 patient after 6 hours including 2 patients who did not require ventilator or isotropic support recovered, rest succumbed to MOF. Patient survival rate was $31.25 \%(\mathrm{n}=5)$ and renal recovery rate was $43.75 \%$ 

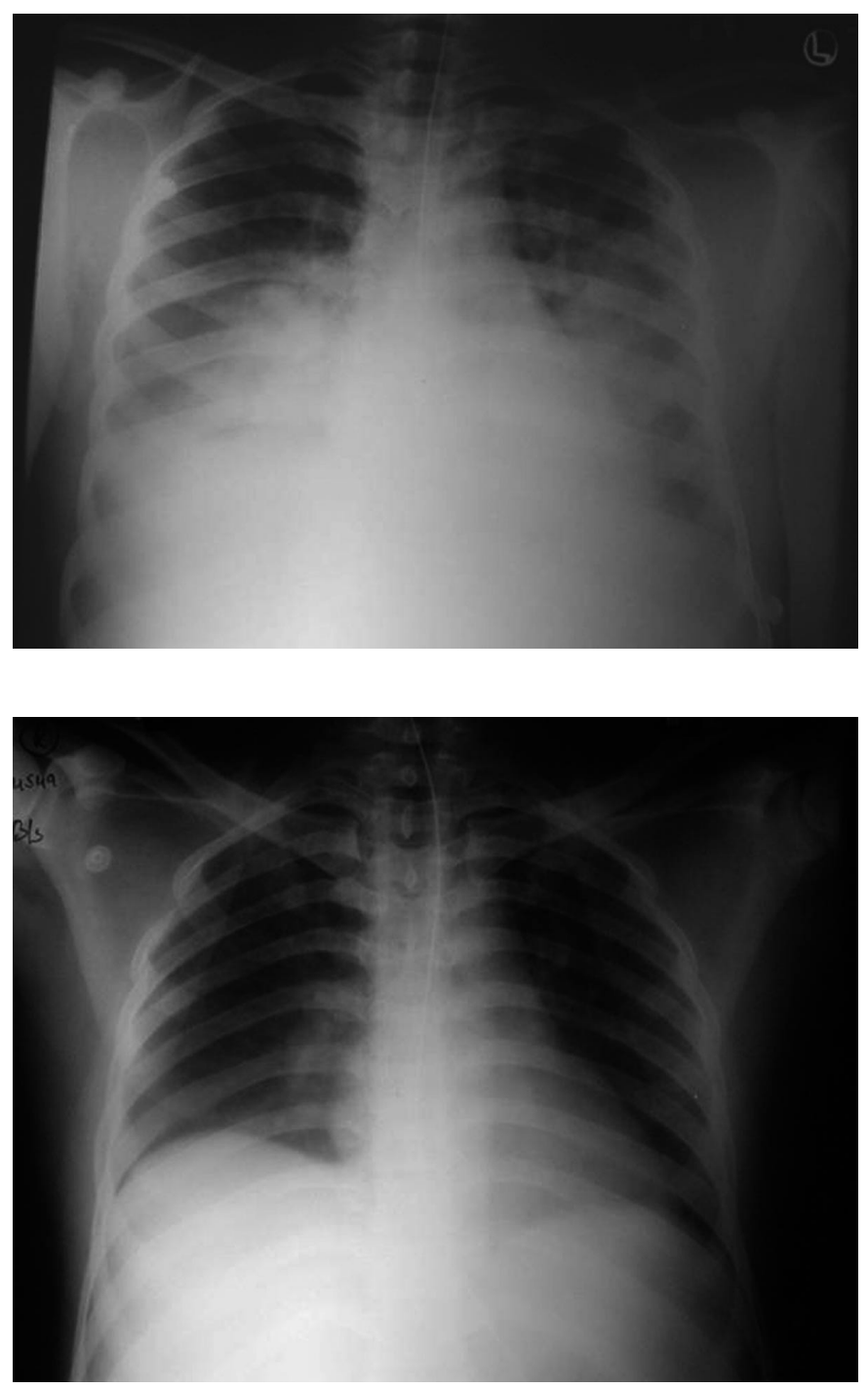

$(n=7)$. No significant complications observed related to procedure. Conclusions: ECBP is safe, simple, adjuvant support therapy in ARDS with a beneficial role over pulmonary and hemodynamic functions, but needs to be initiated early and before requiring the ventilatory or high dose inotropic support for the best outcome.

\section{4}

\section{Continuous Renal Replacement Therapy in a Burn ICU: Launch of a Program}

\section{K.K. Chung, E.A. Mann, E.E. Horvath, B. Stephens, E.M. Renz, S.E. Wolf, J.B. Holcomb \\ U.S. Army Institute of Surgical Research, Texas, USA}

Background: Access to Continuous Renal Replacement Therapy (CRRT) at our institution was previously non-existent. Application of high-volume continuous venovenous hemofiltration (HVCVVH) in patients with multiple organ failure is controversial and not universally considered a proven treatment modality. Severely burned patients have a high mortality with many developing acute renal failure as one component of their multiple system failure. Early initiation of therapies such as CRRT and application of HVCVVH in select patients may be beneficial. Objective: To describe the steps taken to successfully implement an independent CRRT program in a burn ICU. Main: A medical intensivist with credentials to prescribe CRRT was hired by the institution. In addition, a medical consulting firm assisted in product selection and acquisition, development of order sets, flowsheets, nursing and physician standard operating procedures, and assistance with meeting competency standards for all the staff. Two months prior to launch, weekly multi-disciplinary meetings were held to discuss various developmental issues. A month prior to the launch, the consulting firm delivered eight 90 minute introductory didactic sessions reaching approximately $90 \%$ of the ICU staff working various shifts. A week prior to launch, our CRRT machine vendors delivered a week long intensive training camp for 10 'super-users', consisting of both RNs and LVNs, previously identified by the institution. Satisfactory completion of a comprehensive competency checklist along with a grade of $85 \%$ or higher on a proficiency exam were required to be recognized by our institution as a 'super-user'. These 'super-users' were then tasked to train the rest of the nursing staff. Our first patient was successfully treated using our new machine a week later. A case series with lessons learned will be presented. Conclusion: Implementation of a new CRRT program in a previously naive ICU can be a daunting task. With focused application of various resources, along with institutional support, we have successfully initiated this new capability in a Burn ICU.

\section{5}

\section{Hemodiafiltration in Intensive Care Unit: Single Centre Experience from India}

\author{
S.Soni, A.P. Nagrik, A.S. Raman, G.K. Adikey \\ Department of Nephrology, Mediciti Hospitals, \\ Hyderabad, India
}

Introduction and Purpose of the Abstract: The incidence of acute renal failure (ARF) in the hospital setting is increasing. It portend excessive morbidity/mortality and a considerable burden on hospital resources. In developing country like India it presents an excessive burden over limited resources. Extracorporeal therapies are the latest technology to show promise in the management of critically ill ARF. It is said that the potential of such a therapy goes beyond providing solely renal support. Global blood purification and removal of multiple inflammatory mediators. This system is being labelled as MOST (Multiple Organ Support Therapy). However, the available results are contradictory. Aim: To analyse the clinical setting and outcome of critically ill ARF patients managed with continuous renal replacement therapy (CRRT) and to assess utility of SOFA (Sequential Organ Failure Assessment) score in predicting outcome of CRRT. Methodology: From January 2004 to November 2005, 50 hemodynamically unstable cases of ill ARF underwent CVVHDF in intensive care unit. Hemodynamic instability was defined as systolic $\mathrm{BP}<100 \mathrm{mmHg}$ in spite of one or more inotropic agents. SOFA score before initiation of dialysis support was recorded in all cases. SOFA score determines organ 
failure in six systems. Score of $0-4$ is awarded for each system. The total score may vary from 0-24. Higher the score, severe is the disease. Complete renal profile including ultrasound was done in all cases. CVVHDF was performed using Diapact Braun CRRT machine. Vascular access used were - Femoral in 32, Internal Jugular in 8, AVF in 4 and Subclavian 6 patients. 1.7\% P.D. fluid was used as dialysate. $0.9 \%$ NS with addition of $10 \% \mathrm{Ca}$ gluconate, Magnesium sulphate, Sodabicarbonate and Potassium chloride added sequentially in separate units were used for replacement, carefully monitoring their levels. Anticoagulation of extracorporeal circuit was achieved with systemic heparin in 21 and regional heparin in 5 patients. No anticoagulation was used in 24 patients. Results: Of the 50 patients studied, 29 were males and 21 females (1.4:1). The average age was 52.88 yrs (20-75). The co-morbidities included - Type II DM in 29 (58\%), Hypertension - 20 (40\%), Coronary artery disease $-13(26 \%)$ and Chronic kidney disease in $8(18 \%)$ patients. Cause of ARF included Sepsis in $24(48 \%)$, Hemodynamically mediated in $18(36 \%)$ and acute over Chronic renal failue in $8(16 \%)$ patients. The mean pre-dialysis systolic and diastolic BP was 92.28 and $61.19 \mathrm{mmHg}$ respectively. $31(62 \%)$ patients were ventilated. The mean duration of CRRT was 27.51 hours. The mean ultrafiltration rate was $71.80 \mathrm{ml} / \mathrm{hr}$. Pre-dialysis serum creatinine, blood urea, serum bicarbonate and serum potassium levels were $6.05 \mathrm{mg} / \mathrm{dl}$, $126.81 \mathrm{mg} / \mathrm{dl}, 14.94 \mathrm{mEq} / \mathrm{l}$ and $4.94 \mathrm{mEq} / \mathrm{dl}$ respectively. The overall mortality was $74 \%$. Average SOFA score was 14.22 . The variables influencing survival vs. mortality were - serum creatinine (4.31 vs. 6.93 , $\mathrm{p}-0.02)$, blood urea (111.33 vs. $134.56, \mathrm{p}-0.02)$, serum bicarbonate (18.34 vs. 11.96, p -0.05) and SOFA score (11.42 vs. 15.33, p -0.02), while blood pressure, serum potassium, mechanical ventilation and UF rate did not affect outcome. Common complications observed during the therapy included Hypokalemia in 19 (38\%), Hyperglycemia in 17 (34\%) and Hypophosphatemia in 16 (32\%) patients. Conclusions: (1) Despite impressive advances in treating critically ill patients, mortality is dismally high. (2) Multiorgan dysfunction (as assessed by SOFA score) adversely affects outcome of CRRT. (3) SOFA score is useful predictor of outcome. (4) Level of azotemia and severity of metabolic acidosis are important predictors of outcome. (5) Monitoring of electrolytes, specially Potassium, Phosphorous and Sugar is mandatory.

\section{6 \\ Citrate Accumulation Depends on the Dose of Hemofiltration in Patients on CVVHDF with Liver Dysfunction}

\author{
G. Harish Reddy, H. Chaparala, H. Suh, R. Bukovsky, \\ N.K. Wadhwa \\ State University of New York at Stony Brook, NY, USA
}

Background: We had previously reported that regional citrate anticoagulation during continuous veno-venous hemodiafiltration (CVVHDF) does not lead to citrate accumulation in patients with liver dysfunction. The current study evaluated the effect of filtration on citrate accumulation in acute renal failure patients with liver dysfunction who received CVVHDF using citrate as anticoagulant. Methods: We retrospectively reviewed 17 patients who underwent CVVHDF between May 2004 and October 2005. Group A (N = 7; 6 males and 1 female with a mean age of 51 years) received replacement fluid BGK2/0 at $1000 \mathrm{ml} / \mathrm{hr}$ between September 2004 and April
2005. Group B ( $N=10 ; 6$ males and 4 female with a mean age of 65 years) received replacement fluid BGK $2 / 0$ at $1500 \mathrm{ml} / \mathrm{hr}$ between May 2005 and October 2005. Citrate Dextrose-Formula A (ACD-A) was initiated at $150 \mathrm{ml} / \mathrm{hr}$. The rate was adjusted to maintain post filter iCa between $1.0-1.4 \mathrm{mg} / \mathrm{dl}$. Calcium chloride was administered as continuous intravenous infusion to maintain systemic serum $\mathrm{iCa}$ between 4.0 to $4.5 \mathrm{mg} / \mathrm{dl}$. Blood flow rate was $100 \mathrm{ml} / \mathrm{min}$. Prismasate BGK 0/3.5 (Gambro, USA) in 4 patients and Prismasate BGK 4/2.5 in 13 patients was delivered at $500 \mathrm{ml} / \mathrm{hr}$ as a dialysate. Serum and effluent citrate levels were measured daily. Systemic and post filter serum ionized calcium were measured one hour after initiating CVVHDF and then every six hours. Results: Data is summarized in the table. Conclusion: Serum citrate levels are significantly lower in the group receiving higher dose of filtration. Hemofiltration appears to be the major route of citrate elimination in these patients.

\begin{tabular}{lccl}
\hline Variables & $\begin{array}{c}\text { Group A (N =7) } \\
\text { Mean } \pm \text { SD }\end{array}$ & $\begin{array}{l}\text { Group B (N =10) } \\
\text { Mean } \pm \text { SD }\end{array}$ & P value \\
\hline AST (IU/l) & $2130 \pm 3130$ & $2280 \pm 3480$ & $\mathrm{NS}$ \\
Total bilirubin $(\mathrm{mg} / \mathrm{dl})$ & $7.0 \pm 5.05$ & $7.73 \pm 6.1$ & $\mathrm{NS}$ \\
ACD-A (ml/hr) & $117.2 \pm 47.4$ & $143.2 \pm 62.8$ & $\mathrm{NS}$ \\
Pre filter iCa $(\mathrm{mg} / \mathrm{dl})$ & $3.4 \pm 0.32$ & $4.2 \pm 0.61$ & $<0.001$ \\
Post filter iCa $(\mathrm{mg} / \mathrm{dl})$ & $1.7 \pm 0.45$ & $1.6 \pm 0.14$ & $\mathrm{NS}$ \\
Serum citrate $(\mathrm{mg} / \mathrm{dl})$ & $4.8 \pm 2.3$ & $2.0 \pm 1.0$ & $<0.001$ \\
Effluent citrate $(\mathrm{mg} / \mathrm{dl})$ & $59.2 \pm 23.41$ & $70.4 \pm 13.01$ & $\mathrm{NS}$ \\
\end{tabular}

17

\section{Apheresis in Critical Renal Diseases}

N. Chen, P. Shen, P. Zhu, W. Zhang, X. Chen, H. Ren

Ruijin Hospital affiliated to Shanghai Jiaotong University, China

Objective: To observer the effects of plasma exchange (PE) and Immunoabsorbtion (IA) on the critical renal diseases. Method: 67 patients with critical renal diseases were enrolled and treated with PE and IA from 2000 to 2004, including males 31 and females 36, aged from 9 to 84 years $(45.84 \pm 17.13)$. The etiology of diseases included multiply myeloma (MM, $\mathrm{n}=13$ ), lupus nephropathy $(\mathrm{LN}, \mathrm{n}=12)$, thrombotic thrombocytopenic purpura (TTP, $\mathrm{n}=3$ ), hemolytic uremia syndrome (HUS, $n=5$ ), ANCA associated vasculitis (MPA or WG, $n=8$ ), macroglobulinemia $(\mathrm{n}=3)$, fulminant hepatitis $(\mathrm{n}=2)$, anti-GBM syndrome $(n=1)$, erythroderma $(n=1)$ and others $(n=9)$. Results: These patients were treated with single membrane plasma exchange (SPP) 51 patient-times, double filter plasma exchange (DFPP) 123, IA 18, SPP plus DFPP 5 and DFPP plus IA 3. Each patient received these therapies for 3.04 times, 2.9 liter of plasma in average. After the treatment, in the 3 TTP patients the platelet increased from $42.88 \pm 40.48 \times 10-9 / \mathrm{L}$ to $199.86 \pm 41.77 \times 10-9 / \mathrm{L}$ and hemoglobin from $57.63 \pm 32.25 \mathrm{~g} / \mathrm{L}$ to $111.43 \pm 13.25 \mathrm{~g} / \mathrm{L}$; in the HUS patients, the impaired renal function recovered in 5 cases, one patient entered the maintenance dialysis and 3 patients died; the IgM level in all the patients with macroglobulinemia reduced significantly; in all LN (type IV), the titers of anti-ds-DNA antibody and immunoglobulin ( $\mathrm{IgG}$ ) decreased from $27.35 \pm 21.45 \mathrm{mg} / \mathrm{dl}$ to $8.79 \pm 13.97 \mathrm{mg} / \mathrm{dl}$ and from $738 \pm 361 \mathrm{mg} / \mathrm{dl}$ to $438 \pm 262 \mathrm{mg} / \mathrm{dl}$, respectively, and positive ANA in 2 patients turned to negative; in the 
$13 \mathrm{MM}$ patients (11 with type $\operatorname{IgG}$ and 2 with type $\operatorname{IgA}$ ), the levels of IgG and IgA decreases from $4898 \pm 4724 \mathrm{mg} / \mathrm{dl}$ to $2645 \pm 2698 \mathrm{mg} / \mathrm{dl}$ and from $4829 \pm 6625 \mathrm{mg} / \mathrm{dl}$ to $1165 \pm 2430 \mathrm{mg} / \mathrm{dl}$, respectively and hemoglobin increased from $56.0 \pm 3.0 \mathrm{~g} / \mathrm{L}$ to $83.8 \pm 27.9 \mathrm{~g} / \mathrm{L}$, and the level of serum creatinine decreased in 4 patients, no change in 5 patients and increased in one patient; in all the vasculitis patients, the titers of ANCA reduced, and the renal function recovered in one patient, improved in 4 patients and maintenance dialysis in 3 patients. Conclusion: Plasma exchange can treat TTP effectively. PE downregulated the abnormally increase of immunoglobulin, light-chain protein presented in myeloma, furthermore, combined with chemotherapy could attenuate the renal function loss, promoted the survival rate. PE could cleanse the auto-antibody markedly, and decrease the titer of anti-ds-DNA antibody more efficiently. PE is recommended to treat the severe case of LN, LN + APS, TTP/HUS. To the macroglobulinemia patients, we recommend PE or IA combined with chemotherapy in short period. PE and IA have significant effects on the treatment of TTP/HUS, MM, macroglobulinemia, critical lupus nephropathy, but the future effects need to be further investigated.

\section{8 \\ Evaluation of Different Blood Purification Modes in Acute Renal Failure (ARF)}

W. Zhang, Y. Xu, H. Shi, J. Xie, X. Feng, N. Chen

Shanghai Rui Jin Hospital affiliated to Shanghai Jiao Tong University, China

Objective: To applicate different techniques of blood purification in ARF, and thus to improve the outcome and level of medicare. Methods: Among 191 ARF patients undergoing blood purification, we assessed corresponding prognosis in different modes such as intermittent HD (iHD), continuous veno-venous hemofiltration (CVVH), slow low extended dialy dialysis (SLEDD), continuous ambulatory peritoneal dialysis (CAPD), and CVVH combined with iHD in different types of ARF. Results: One hundred and ninety-one ARF patients were undergoing blood purification, which was accounting for $34.6 \%$ of total ARF during the same period. Among them, 120 males and 71 females with mean age of $49.14 \pm 19.61 \mathrm{yrs}$, serum urea level of $24.35 \pm 14.31 \mathrm{mmol} / \mathrm{L}$, serum creatine levels of $533.98 \pm$ $397.21 \mathrm{umol} / \mathrm{L}$. The average clearance of creatine was $17.61 \pm 5.43 \mathrm{~L} /$ $24 \mathrm{~h}$ according to the C-G formula. Dialysis moduality included 101 on iHD, 57 on CVVH and SLEDD associated with PE (14) and IA (3), 16 on CAPD. The indications for CVVH or SLEDD included complicated ARF such as acute rhabdomyolysis syndrome, severe systemic vasculitis, congestive heart failure with unstable circulating blood volume, hypotensive shock, encephalic edema, ARDS, hypercalcemia crisis, SIRS, acute pancrititis complicated ARF, maliganancy, ARF after cardiac operation, hepatic encephalopathy and liver transplantation with multiple organ dysfunction syndrome (MODS). Pre-dilution CVVH was conducted with $150-200 \mathrm{ml} / \mathrm{min}$ blood volume, once per day or every other day until the recovery phase or transferring to maintenance $\mathrm{HD}$, the replacement fluids were $4 \sim 6$ liters per hour, the treatment time were $8 \sim 12$ hours, with Fresenius F60 filter. The combination of $\mathrm{CVVH}$ and $\mathrm{HHD}$ were given in liver transplantation and hepatic encephalopathy especially with hyperbilirubinemia. The survival rate of CVVH was $35 / 57$ (61.4\%), the mortality was $38.6 \%$ with 5 hemorrhage and 4 hypotesion. iHD were performed only in those ARF without complications, the frequency was every other day, the treatment time was 5 hours, the survival rate was 70/101 (69.3\%), 25 were died and 6 were lossing follow-up (30.7\%). CAPD were performed in Child and elderly patients. Among 16 CAPD patients, the recovery rate was $31.3 \%$, the mortality $43.8 \%$, and $24.9 \%$ lost followup. The total outcome of 191 ARF showed 47.1\% with recovery $18.8 \%$ transferring to maintenance hemodialysis, $30.9 \%$ death and 3.2\% lost follow-up. Conclusions: (1) In ARF, dialysis should be started early. Intensive and early dialysis plays a key role in the prognosis of ARF. (2) Considering with the efficacy of CRRT and iHD, data from ARF prognosis showed no significance. It is recommended to use CRRT or SLEDD in complicated ARF. (3) The combination of CRRT and iHD is prefer to certain types of ARF such as hepatic encephalopathy, liver transplantation, acute rhabdomyolysis syndrome and hyperbilirubinemia. (4) Whatever which technique choosen, the dialysis adequacy is more important to achieve to correct the endoenvironment disturbance and to improve the renal recovery as soon as possible.

19

\section{Different Actions of Inflammatory Mediators and Growth Factors on Tubular Cells (TC): Role in the Recovery from Acute Kidney Injury (AKI) and Potential Diagnostic and Therapeutic Applications}

\author{
V. Cantaluppi, A. Pacitti, G. Podda, L. Biancone, \\ G.P. Segoloni, G. Camussi \\ Nephrology, Dialysis and Renal Transplantation Unit, \\ University of Torino, Italy
}

The number of patients with AKI in ICUs has been sensitively increasing in a clinical setting characterized by injury of TC in presence of sepsis or SIRS. During 2000-2005, we treated about 130 AKI patients/year performing more than 1300 CRRT sessions/year, with a prevalence of 'systemic inflammation' of more than $40 \%$. Basing on these considerations, we evaluated the different effects of inflammatory mediators and growth factors on TC. We previously demonstrated the presence of small peptides in the urine of critically ill patients due to fragmentation of filtered proteins. We have now extended these findings to more than $50 \mathrm{AKI}$ patients confirming that the increase of urinary fragments is mediated by the up-regulation of megalin on TC surface and is an indicator of a 'pre-renal' state. The increased expression of megalin may be related to a sort of defense state against malignant inflammation for maintaining TC viability. However, the prolonged incubation with cytokines or with sera derived from patients with fragmented proteins resulted in alteration of tubular polarity, selective permeability, adhesion to extracellular matrices and triggering of apoptotic pathway throughout Fas and caspases activation. We also studied the key role of TC in the release of different mediators and growth factors that promote de-differentiation, proliferation, migration and tissue repair. Our findings underline the importance of new therapies of blood purification aimed to remove inflammatory mediators, confirm the diagnostic use of fragmented urine proteins and may have potential applications in the engineering of TC or kidney derived stem cells for bioreactors. 
20

\section{Urinary KIM-1 Predicts Severe AKI Earlier than Serum Creatinine in Pediatric Patients}

\author{
A. Akcan-Arikan ${ }^{1}$, W. Han ${ }^{2}$, K. Washburn ${ }^{1}$, L.L. Loftis ${ }^{1}$, \\ S.L. Goldstein', J.V. Bonventre ${ }^{2}$ \\ ${ }^{1}$ Baylor College of Medicine, Houston, TX, ${ }^{2}$ Harvard Medical \\ School, Boston, MA, USA
}

Acute kidney injury (AKI) is common in critically ill patients (pt) and carries a high mortality. Standard methods of detecting AKI such as serum creatinine ( $\mathrm{SCr}$ ) may not become abnormal until substantial kidney damage has occurred, as recent adult and pediatric study shows 'small' $\mathrm{SCr}$ increases $(>0.3 \mathrm{mg} / \mathrm{dL})$ are associated with increased mortality. Kidney injury molecule-1 (KIM-1) has been isolated from urine in humans with AKI and is an early AKI marker that precedes SCr changes in small animal models. We prospectively studied critically ill (ventilated and/or receiving $1+$ inotrope) pediatric pt to assess if KIM-1 precedes SCr rise in AKI. Daily urine samples were collected on day 1 to 4 of ICU admission for KIM-1 analysis. AKI severity was determined using pediatric modified RIFLE criteria (Risk, Injury, and Failure). The primary outcome was highest RIFLE level achieved by ICU day 14 (RIFLEmax). 102 pt (56 M, 46 F, mean age $5.5 \pm 5.9$ years) were studied. $44 \mathrm{pt}(43 \%)$ did not develop AKI (control), 58 patients $(57 \%)$ developed AKI $(\mathrm{R}=42, \mathrm{I}=11$, and $\mathrm{F}=5$ ). The mean KIM-1 level in the urine on day 1 was $0.28 \pm 0.65$ in the control group; this level was increased almost 10 fold on day 1 and over 20 fold by day 4 in the RIFLE-F group (see table), one-way ANOVA $3 / 5 \mathrm{~F}$ pt had elevated KIM-1 levels on day 1; all $\mathrm{F}$ pt had elevated KIM-1 levels by day 4. Urine KIM-1 excretion preceded rising $\mathrm{SCr}$ in $4 / 5 \mathrm{~F}$ pt. Our data support the concept that urinary markers can be used to detect AKI earlier than SCr rise, and hopefully will allow for earlier intervention to prevent worsening AKI and/or lessen mortality/morbidity in critically ill pt with AKI.

\begin{tabular}{lll}
\hline & Day 1 & Day 4 \\
\hline Control & $0.28 \pm 0.65$ & $0.20 \pm 0.33$ \\
Risk & $0.29 \pm 0.92$ & $0.12 \pm 0.41$ \\
Injury & $0.15 \pm 0.35$ & $0.12 \pm 0.30$ \\
Failure & $2.60 \pm 2.62^{*}$ & $4.46 \pm 6.68^{*}$ \\
\hline
\end{tabular}

${ }^{*} \mathrm{p}<0.001$.

\section{1 \\ Evaluation of the RIFLE Criteria in Critically III Children with Acute Kidney Injury}

\section{A. Akcan-Arikan, K. Washburn, L.L. Loftis, \\ C. Kennedy, L.S. Jefferson, S.L. Goldstein \\ Baylor College of Medicine, Houston, TX, USA}

Background: Outcome studies for patients with acute kidney injury (AKI) suffer from lack of a consistent AKI definition. Recently, RIFLE (Risk, Injury, Failure, Loss, ESRD) criteria were proposed to standardize the degree of AKI in adults based on creatinine clearance and urine output. Objective: We prospectively studied a pediatric modified RIFLE in 102 critically ill pediatric (ped) patients (pts) over 7 months to assess (1) AKI incidence and course using RIFLE and (2) if AKI by RIFLE predicted renal function course and/or non-renal comorbidities. Design/Methods: See table. Results: Mean pt age was 5.5, 5.8, 46 F and 56 M. 44/102 (43\%) did not develop AKI, $58 \mathrm{pts}$ $(57 \%)$ reached $R(n=42), I(n=11)$ or $F(n=5) .3$ patients $(2.9 \%)$ required renal replacement therapy as CVVHD and 9 pts died. Severity of illness as measured by Ped Risk of Mortality (PRISM) score was higher for AKI pts (15.2) vs. non AKI pts $(11.8, \mathrm{p}<0.05)$. The majority of AKI patients $(60 \%, \mathrm{n}=35)$ developed AKI within 7 days of ICU admission. Pts without AKI by RIFLE in the first 7 days were significantly less likely to develop worsening AKI later (2/67 vs. 14/35, $\mathrm{p}<0.001$; NPV 97\%). Pts who developed AKI after 7 days did not progress to F. AKI was not more prevalent in pts with preexisting conditions. Length of stay (hospital and ICU) and mechanical ventilation did not differ for AKI vs. no AKI. Conclusions: Our data show (1) a majority of critically ill ped pts develop AKI by RIFLE. (2) Pts who develop AKI do so early in the ICU course. (3) Pts who do not develop AKI early are unlikely to develop severe AKI. We conclude that the RIFLE criteria may serve well to characterize the pattern of AKI in critically ill ped pts. Further study is required to correlate degree of AKI by RIFLE with short and long term pt outcome.

\begin{tabular}{lll}
\hline & \multicolumn{2}{l}{ Pediatric modified RIFLE criteria } \\
\cline { 2 - 3 } & Estimated CrCl & Urine output \\
\hline Risk & GFR decrease by $25 \%$ & $<0.5 \mathrm{ml} / \mathrm{kg} / \mathrm{hr}$ for 8 hours \\
Injury & GFR decrease by $50 \%$ & $<0.5 \mathrm{ml} / \mathrm{kg} / \mathrm{hr}$ for 16 hours \\
Failure & GFR decrease by $75 \%$ or & $<0.3 \mathrm{ml} / \mathrm{kg} / \mathrm{hr}$ for 24 hours \\
& GFR $<35 \mathrm{ml} / \mathrm{min} / 1.73 \mathrm{~m}$ & or anuric for 12 hours \\
\hline
\end{tabular}

\section{2}

Renal and Hemodynamic Effects of Intrarenal Nesiritide in Heart Transplant Patients

\author{
V.S. Mathur', J.T. Heywood ${ }^{2}$, A. Ho ${ }^{3}$
}

${ }^{1}$ MathurConsulting, Woodside, CA, ${ }^{2}$ Scripps Clinic, La Jolla, CA, ${ }^{3}$ Loma Linda Medical Center, Loma Linda, CA, USA

Purpose: Inferring the renal effects of drugs in trials of decompensated heart failure using serum creatinine only or retrospective analyses are potentially confounded by a myriad of factors, including concomitant medication use and volume status. Our goal was to determine the actual renal effects of nesiritide (NES), B-type natriuretic peptide, using gold standard methodology and also to assess to what extent the blood pressure lowering effects of NES contribute to its renal effects. Methods: We conducted an open-label prospective trial in 10 stable patients post-heart transplant with a history of cyclosporine or tacrolimus use and scheduled for routine left and right heart catheterization. Intra-renal (IR) NES $(0.01 \mathrm{mcg} / \mathrm{kg} / \mathrm{min}$ infusion) was administered for 30 minutes (with $(\mathrm{n}=4)$ or without $(\mathrm{n}=6)$ a $2 \mathrm{mcg} / \mathrm{kg}$ IR bolus) via a commercially available bifurcated renal infusion catheter (Benephit Infusion System, FlowMedica) prior to coronary angiography. Results: The patients were $3.0 \pm 1.0$ years post heart transplantation. 
The mean age was $56.2 \pm 3.9$ years, $50 \%$ were male, and baseline characteristics included: serum creatinine, $1.4 \pm 0.1 \mathrm{mg} / \mathrm{dL}$; serum BNP, $111.6 \pm 30.1 \mathrm{pg} / \mathrm{mL}$; ejection fraction $69 \pm 1.2 \%$. Systemic exposure to NES, as assessed by plasma BNP levels was significantly higher when NES was administered as an IR bolus $(2 \mathrm{mcg} / \mathrm{kg})$ with infusion $(0.01 \mathrm{mcg} / \mathrm{kg} / \mathrm{min})$ vs. infusion alone $(\mathrm{p}=0.016)$. These data suggest that the renal excretion and metabolism of NES can be overwhelmed by a large IR bolus, resulting in greater systemic spillover. IR NES infusion did not affect blood pressure, consistent with a renal first pass elimination effect. In contrast, blood pressure declined following an IR NES bolus. Glomerular filtration rate (GFR, inulin clearance) increased significantly from a baseline of $45.6 \pm 2.2 \mathrm{ml} / \mathrm{min}$ by $+12.0 \%$ within 30 minutes of the start of IR NES $(p=0.005)$. In patients not receiving a bolus, the effects tended to be greater $(+13.4 \%)$ vs. those receiving a bolus $(+9.9 \%)$. Renal plasma flow (RPF, paraaminohippurate clearance) increased significantly from a baseline of $297.8 \pm 20.2 \mathrm{ml} / \mathrm{min}$ by $+16.4 \%$ within 30 minutes of the start of IR NES $(p=0.004)$. In patients not receiving a bolus, the effects tended to be greater $(+20.7 \%)$ vs. those receiving a bolus $(+11.2 \%)$. Renal vascular resistance declined by $25.6 \%$ within 30 minutes of initiation of IR NES $(p=0.005)$. Cardiac hemodynamics were not significantly affected during IR NES infusion in these euvolemic patients. Bilateral renal artery cannulation was rapidly accomplished in all patients (77.5 \pm 31.3 seconds) and no procedural complications occurred. There were no drug-related adverse events. Conclusions: IR NES safely, rapidly, and significantly increases the GFR and RPF in stable heart transplant patients with renal insufficiency and decreases renal vascular resistance. The fact that RPF increased more than GFR suggests that there is relatively greater afferent than efferent arteriolar dilatation. IR delivery of NES may be a useful strategy for optimizing the drug's beneficial renal hemodynamic effects without causing hypotension. As such, IR NES may prove to be a therapeutic strategy for reversal of renal insufficiency in patients with acute heart failure or those with acute kidney injury in a variety of settings.

\section{3}

Intra-renal Nesiritide Rapidly and Significantly Improves the Glomerular Filtration Rate and Renal Plasma Flow in Patients with Chronic Kidney Disease Receiving Contrast Media: Data from a Multicenter, Randomized, Placebo-controlled Trial

\author{
V.S. Mathur ${ }^{1}$, M. Price ${ }^{2}$, L. Guzman ${ }^{3}$, P. Teirstein ${ }^{2}$ \\ ${ }^{1}$ MathurConsulting, Woodside, CA, ${ }^{2}$ Scripps Clinic, \\ La Jolla, CA, ${ }^{3}$ University of Florida - Jacksonville, \\ Jacksonville, FL, USA
}

Purpose: Previous attempts to demonstrate clinical efficacy of atrial natriuretic peptide in acute kidney injury may have been thwarted by negation peptide's renal vasodilatory effects by doserelated hypotension. We hypothesized that intra-renal (IR) delivery of nesiritide (NES) would both increase renal effects as well as diminish hypotension due to renal first pass elimination of drug, thereby widening the renal therapeutic window. We are conducting a multicenter, randomized, controlled trial to determine the role of targeted renal therapy with NES in preventing vasoconstriction from contrast media. Methods: Patients $(\mathrm{n}=30)$ with creatinine clearance $<80 \mathrm{cc} /$ min are randomized 1:1:1 to IV NES, IR NES, or placebo $\times 30$ minutes prior to angiography and during angiography. Glomerular filtration rate (GFR) and renal plasma flow (RPF) were assessed using insulin and para-aminohippurate. The Benephit System (FlowMedica), used for simultaneous bilateral drug delivery, is a commercially available device consisting of an $8 \mathrm{~F}$ or $5 \mathrm{~F}$ sheath through which a bifurcated renal infusion catheter is placed. The $8 \mathrm{~F}$ sheath accommodates simultaneous placement of a $<6 \mathrm{~F}$ catheter. Results: We report interim data from this trial $(n=16)$. Systemic exposure to NES, as assessed by plasma BNP levels, was double when the drug was administered IV vs. IR $(\mathrm{p}=0.03)$. RPF increased by a maximum of $+5.4 \%,+17.0 \%$, and $+28.6 \%$ in the placebo, IV, and IR NES groups, respectively. From a baseline of $50.5,49.7$, and $55.3 \mathrm{ml} / \mathrm{min}$, respectively, the GFR increased by a maximum of $+7.6 \%,+18.0 \%$, and $+30.0 \%$ in the placebo, IV, and IR NES groups, respectively ( $p<0.05$ for IV and IR NES). Despite receipt of a large volume of contrast $(\sim 260 \mathrm{ml})$, improvements in RPF and GFR noted during IV and IR NES infusion were maintained at least 2 hours following drug discontinuation. The magnitude of the aldosterone suppression was greater during IR NES than IV NES. Bilateral renal artery cannulation was rapidly accomplished (mean 164 seconds) and no procedural complications occurred in patients receiving the Benephit Infusion System. Maximal systolic blood pressure reduction in patients receiving IR vs. IV NES was $-10.7 \pm 10.1 \mathrm{mmHg}$ vs. $-23.7 \pm 6.6 \mathrm{mmHg}$. Conclusions: Nesiritide rapidly, robustly, significantly, and safely increases GFR and RPF in vasculopathic patients with chronic kidney disease. The magnitude of these effects is approximately twice as great when the drug is administered IR vs. IV. For example, GFR increased by $30 \%$ within 30 minutes of IR NES $(0.02 \mathrm{mcg} /$ $\mathrm{kg} / \mathrm{min}$ ). Our data suggest that NES actually has direct renal vasodilatory effects as we have shown that the drug can improve RPF in stable patients with chronic kidney disease who have no reason clinically to have renal vasoconstriction. Compared to IV NES, treatment with IR NES is associated with $\sim 50 \%$ lower systemic blood levels and $\sim 50 \%$ less systemic blood pressure lowering, consistent with our hypothesis that targeted renal treatment may be able to avert limiting systemic adverse effects of NES. Our data suggest that IR NES may have a role in the treatment and prevention of ischemic acute kidney injury.

\section{Technique Characteristics}

\section{4 \\ Continuous Veno-venous Hemodiafiltration Therapy for Acute Decompensation with Cerebral Edema in Maple Syrup Urine Disease}

J.J. Zaritsky, J.A. Martinez, O. Yadin

David Geffen School of Medicine, Mattel Childrens Hospital, UCLA, Los Angeles, USA

Maple syrup urine disease (MSUD) is caused by an inheritable deficiency in 2-ketoacid dehydrogenase and results in the systemic 
accumulation of branched chained amino acids (BCAAs) and their metabolites. During episodes of metabolic decompensation characterized by extremely high serum levels of BCAAs, affected children have severe neurological deterioration often accompanied by life-threatening cerebral edema. Because endogenous renal clearance of BCAAs is limited, therapy has focused on the rapid extracorporeal removal of BCAAs often via intermittent hemodialysis (IHD). However the rapid diffusive clearance of IHD may lead to solute disequilibrium potentially worsening cerebral edema. In this report, a symptomatic 7 year old male with MSUD who developed cerebral edema underwent CVVHD with a small molecule clearance rate of $37.5 \mathrm{ml} / \mathrm{min} / \mathrm{m}^{2}$. This approach resulted in a nearly $50 \%$ decrease in BCAA levels within the first 4 hours of treatment. Therapy was discontinued at 16 hours due to the rapid improvement in neurological status. At that time, serum leucine levels had fallen from 1581 to $268 \mu$ moles/l, isoleucine levels had fallen from 313 to $110 \mu$ moles/l and valine levels had fallen from 652 to $83 \mu \mathrm{moles} / \mathrm{l}$. Leucine, isoleucine and valine clearance rates measured during the last four hours of therapy were 30.4, 30.9 and $30.7 \mathrm{ml} / \mathrm{min}$ respectively. This case demonstrates that CVVHD is an effective and safe therapy of severe acute decompensation in MSUD accompanied by cerebral edema.

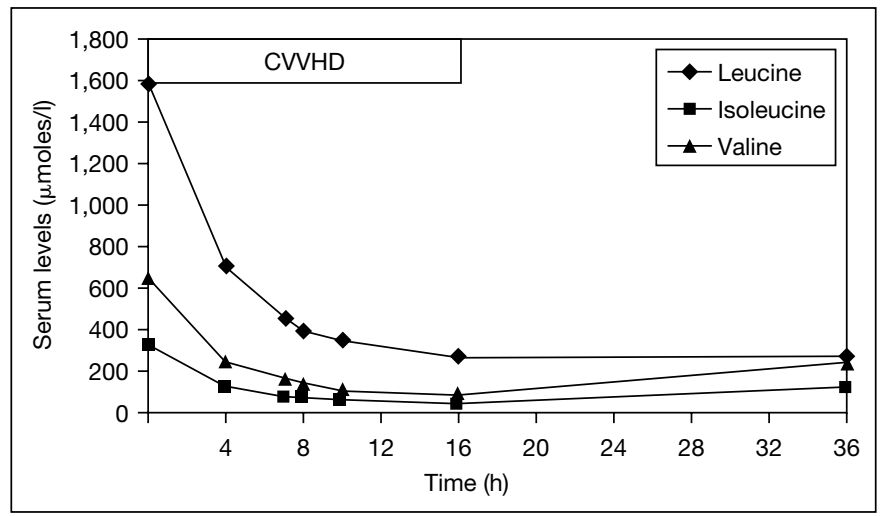

25

\section{Application of CRRT without Anticoagulation in Patients with Bleeding Disorders}

Q. Jin

Renal Division, First Hospital, Peking University, Beijing, China

Objective: To study the feasibility of continuous renal replacement treatment (CRRT) with non-anticoagulation in critical ill patients with high risk of bleeding. Methods: A total of 12 critical ill patients with bleeding diseases requiring continuous renal replacement treatment were prospectively studied. The patients were performed SLEDD (slow low efficient daily dialysis) with total of 28 sessions, the dialyzer were hemophan membrane (GFS Plus-12, Gambro). All circuits received $200 \mathrm{mg}$ heparin during rinsing stage for 30-60 minutes. Before connection of the circuit to the patients, the heparin prime was flushed away with normal saline. During SLEDD, no normal saline was returned to the circuits for flushing. The levels of serum creatinine, serum urea, hematocrit, serum electrolyte and activated clotting time (ACT) before and after CRRT were compared. Results: In the 28 SLEDD, the blood flow was $150-200 \mathrm{ml} / \mathrm{min}$, and the dialysate flow was $3000-4000 \mathrm{ml} / \mathrm{hr}$. The ultrafiltration rate was $200-550 \mathrm{ml} / \mathrm{hr}$. The levels of ACT, serum potassium, serum creatinine and urea after CRRT were decreased significantly $(\mathrm{p}<0.01)$. But the level of serum sodium, chloride, and bicarbonate were not changed significantly $(p>0.05)$. The spontaneous clotting rate in circuit or in dialyzer was $32.1 \%$. But the mean spontaneous clotting time was $9.15 \pm 2.49 \mathrm{hrs}$. Mean CRRT time was $9.33 \pm 2.75 \mathrm{hrs}$. Conclusion: SLEDD without anticoagulation is feasible in patients with bleeding diseases. It is associated with a safe ACT, a satisfactory clearance of toxin and a satisfactory correction of disturbed electrolytes and fluid retention. The circuit longevity is acceptable.

\section{6 \\ Dermatan Sulphate: An Alternative to Heparin for Anticoagulation in CRRT \\ C. Vitale ${ }^{1}$, C. Verdecchia ${ }^{2}$, C. Bagnis ${ }^{1}$, M. Ganzaroli², \\ ${ }^{1}$ Nephrology Unit, Mauriziano Hospital, ${ }^{2}$ Cardiovascular \\ Intensive Care, Mauriziano Hospital, Turin, Italy}

Dermatan Sulphate (DS) is a natural glycosaminoglycan with a unique mechanism of action on the coagulation system. Unlike heparin, DS selectively inhibits thrombin, does not inhibit Factor Xa, is effective also on fibrin-bound thrombin and does not interfere with platelet functions. To date, no data are available on the use of DS with CRRT. In this work, we compared the effects of both DS and heparin as anticoagulant with patients on CRRT because of acute renal failure complicating cardiovascular surgery. In all patients, CRRT was made according to same protocol: Machine, Gambro Prisma ${ }^{\circledR}$; filter, M100 (AN69, $0.9 \mathrm{~m}^{2}$ ); QB, $150 \mathrm{ml} / \mathrm{min}$; QD, $2000 \mathrm{ml} / \mathrm{hr}$; QInfusate, $500 \mathrm{ml} / \mathrm{min}$. With 70 CRRT sessions, DS was used as anticoagulant (DS-CRRT): $150 \mathrm{mg}$ bolus at the start, followed by i.v. infusion of $15 \pm 4.5 \mathrm{mg} / \mathrm{hr}$. With 93 CRRT sessions, anticoagulation was performed with heparin (H-CRRT): starting bolus of $2000 \mathrm{U}$, followed by $634 \pm 292 \mathrm{U} / \mathrm{hr}$. Blood samples for INR, APTT, Fibrinogen, Hb, PLTS were taken at the beginning of CRRT (Basal) and every 8-12 hours, until the filter clotting. The average value of the blood analyses performed over each session was used for statistics. The duration of each CRRT session represented the filter lifetime, namely, the time measured from CRRT start to filter clotting. There were no differences between DS-CRRT and H-CRRT as far as Basal values of aPTT (44.5 \pm 10.8 vs. $41.0 \pm 7.5 \mathrm{sec}, \mathrm{p}=\mathrm{ns})$, INR $(1.40 \pm 0.47$ vs. $1.49 \pm 0.25, \mathrm{p}=\mathrm{ns})$, Fibrinogen $(472 \pm 165$ vs. $416 \pm 152 \mathrm{mg} / \mathrm{dl})$ and $\mathrm{Hb}(9.3 \pm 1.1 \mathrm{vs}$. $9.7 \pm 1.3 \mathrm{~g} / \mathrm{dl}, \mathrm{p}=\mathrm{ns})$ are concerned. Filter lifetime was longer with DS-CRRT than with H-CRRT $(49 \pm 22$ vs. $39 \pm 17 \mathrm{hr} /$ session, $\mathrm{p}<0.01)$. APTT was shorter through DS-CRRT than through $\mathrm{H}-$ CRRT $(55.5 \pm 10.8$ vs. $61.5 \pm 19.5 \mathrm{sec}, \mathrm{p}<0.01)$. PLTS count did not change through DS-CRRT $(144 \pm 101$ vs. Basal $154 \pm 102$, $\mathrm{p}=\mathrm{ns})$, whereas lowered through H-CRRT $(117 \pm 64$ vs. Basal $154 \pm 92, \mathrm{p}<0.01)$. In conclusion, DS allowed higher filter lifetime than heparin, together with lower aPTT levels and without significant reduction of PLTS count. These results suggest that DS can be held as a reliable alternative to heparin as anticoagulant for CRRT in patients with high risk of bleeding after major cardiovascular surgery. 
27

Use of Arterio-venous Graft or Fistula in End Stage Renal Disease Patients Receiving Continuous Renal Replacement Therapy

\section{M.J. Longobucco, R. Barnett, N.K. Wadhwa, R.M. Bukovsky \\ Stony Brook University Hospital, New York, USA}

Continuous Renal Replacement Therapy (CRRT) is increasingly used in end stage renal disease (ESRD) patients with multi-organ failure admitted to intensive care units (ICU). Central venous access is premium in these patients. The present study investigated the use of pre existing arterio-venous (AV) graft or fistula for blood access to perform CRRT in patients with ESRD. The purpose of the study was to evaluate safety and efficacy of the pre-existing AV access in ESRD patients as an alternative to dual lumen catheter for CRRT. Methods: The AV access was used in seven ESRD patients for CRRT from Dec. 2004 through Nov. 2005. All patients had AV access cannulated by a dialysis nurse utilizing $14^{\prime \prime}$ gauge angio-catheters for arterial access and $16^{\prime \prime}$ gauge angio-catheters for venous access. Cannulas were attached to $6^{\prime}$ anesthesia extension tubing which was primed with normal saline (NS). Insertion site was covered with a bio-occlusive dressing. Therapy was immediately initiated after cannulation. An ICU nurse was responsible for monitoring, maintenance and unscheduled discontinuation of therapy. The ICU nurses were educated to monitor the AV access on a $1: 1$ basis and instructed to flush the extension tubing with $10 \mathrm{ml}$ of NS and to clamp the lines in the event that therapy was interrupted. The dialysis nurse was then informed to remove the catheters. Results: Seven ESRD patients, 5 males and 2 females with a mean age of 66 years (range 46-76 years) underwent CRRT using AV access. All patients were intubated and were on vasopressors. Blood flow rate was $100 \mathrm{ml} / \mathrm{min}$. PRISMASATE BGK 4/2.5 (Gambro, USA) was delivered at $500 \mathrm{ml} / \mathrm{hr}$ as a dialysate. PRISMASATE BGK $4 / 0$ was infused at $1500 \mathrm{ml} / \mathrm{hr}$ as replacement fluid. Mean duration of CRRT was 54.75 hours. Citrate anticoagulation was used in $6(86 \%)$ patients. Six (86\%) AV accesses were functioning after the CRRT was discontinued. One patient clotted the AV access. No AV access bleeding, infection or technical problems were observed. Conclusions: Use of AV graft or fistula with an angio-cathether in ESRD patients receiving CRRT is safe and reduces the need for another central venous access.

\section{8}

\section{Regional Citrate Anticoagulation for Predilutional On-line Hemofiltration}

\section{J. Buturovic-Ponikvar, J. Gubensek, N. Skofic, R. Ponikvar \\ Department of Nephrology, University Medical Center, Ljubljana, Slovenia}

Introduction: Intermittent predilutional on-line hemofiltration (HF) could have some advantages as a dialysis method for patients in ICU. In these patients regional citrate anticoagulation is frequently required. The aim of our study was to report our experience with citrate anticoagulation for intermittent predilutional on-line HF. Patients and Methods: 18 patients with acute renal failure in ICU with active bleeding or high bleeding risk were included in this retrospective analysis.
Patients were $71 \pm 10$ years old, 12 were male and 6 were female. 37 online HF procedures with citrate anticoagulation were performed by our protocol. We used $4 \%(0.136 \mathrm{~mol} / \mathrm{l})$ trisodium citrate infused at the arterial line, on-line prepared infusate with $1.25 \mathrm{mmol} / \mathrm{l}$ calcium, lowered sodium $(138 \mathrm{mmol} / \mathrm{l})$ and bicarbonate $(28 \mathrm{mmol} / \mathrm{l})$ concentrations and $1 \mathrm{M}$ calcium chloride infused at the venous line. On-line HF was performed using Gambro AK-200 Ultra S monitor with individual reverse osmosis. Results: Duration of HF treatments was $5 \mathrm{~h} 8 \pm 47 \mathrm{~min}$, infusate volume reached was $78 \pm 81$. Dual-lumen or two single-lumen catheters were used for vascular access, locked with $30 \%$ citrate in interdialysis period. Average blood flow was $269 \pm 52 \mathrm{ml} / \mathrm{min}, 4 \%$ sodium citrate infusion rate was $384 \pm 36 \mathrm{ml} / \mathrm{h}(2.5 \pm 0.3 \%$ blood flow $)$ and $1 \mathrm{M}$ calcium chloride infusion rate was $7.5 \pm 1.3 \mathrm{ml} / \mathrm{h}$. None of the circuits clotted. No severe side effects were noted. Ionized calcium was stable during the procedure $(1.02 \pm 0.16 \mathrm{mmol} / 1$ before and $1.13 \pm$ $0.09 \mathrm{mmol} / \mathrm{l}$ after $\mathrm{HF})$ as was sodium $(141.1 \pm 4.8$ vs. $143.3 \pm$ $3.5 \mathrm{mmol} / \mathrm{l}), \mathrm{pH}(7.36 \pm 0.13$ vs. $7.40 \pm 0.08 \mathrm{mmol} / \mathrm{l})$ and bicarbonate $(24 \pm 4.9$ vs. $26.3 \pm 3.5 \mathrm{mmol} / \mathrm{l})$. Conclusions: Predilutional on-line HF with regional citrate anticoagulation using standard, calcium containing infusate $(1.25 \mathrm{mmol} / \mathrm{l})$ can be safely performed with good anticoagulation and no severe metabolic derangement.

29

\section{Machine Generated Bicarbonate Dialysate for Continuous Therapy: A 10-year Experience}

\author{
B.W. Teo, S. Demirjian, E. Wright, E.P. Paganini \\ Cleveland Clinic Foundation, Cleveland, OH, USA
}

Background: In 1995 we described adapting an intermittent hemodialysis (HD) machine to produce a bicarbonate-based dialysate for use in continuous renal replacement therapies (CRRT). This technique allows for customization of the electrolyte composition of dialysate. Hence, we have almost exclusively supported our acute renal failure (ARF) patients in the intensive care units (ICU) with this dialysate on CRRT in the HD mode. Objective: Describe the clinical, biochemical, operational, and cost-related issues involved with selfgenerated dialysate. Methods: We abstracted data from the ARF support registry for patients who developed ARF in the ICU from 1995-2001, and were initially supported with bicarbonate dialysate CRRT. The registry is a prospective, observational cohort study that captured demographic, dialysis therapy, laboratory, and outcome data. All supported ARF patients were recorded from 1995-1998, and then 1 in 5 patients from 1999-2001. We reviewed monitoring tests for the bicarbonate dialysate, limulus amebocyte lysate assays for bacterial endotoxin; and records of CRRT, discarded dialysate, and cost data. Results: From 1994-2004, we performed an average of $1353 \pm 619$ per year of CRRT (1292 $\pm 587 \mathrm{HD})$. Dialysate delivery from prescription to patient was $30 \mathrm{~min}$ or less for all formulations. There were 405 registry patients. Baseline characteristics: age $59.57 \pm 14.41$ years, weight $84.2 \pm 24 \mathrm{~kg}$, male $65.3 \%$, chronic kidney disease $33.9 \%$, surgery before renal consult $87.9 \% .88 .4 \%$ had veno-venous HD initially and $32.8 \%$ switched therapies. $15.1 \%$ used heparin. The mean dose of dialysis $(\mathrm{ml} / \mathrm{kg} / \mathrm{hr})$ for: day 2 was 14.8 (95\% CI 14.1-15.5) overall, in males 14.0 (13.2-14.8), in females 16.3 (15.0-17.7); 3-day average was 13.2 (12.6-13.8), in males 12.5 (11.9-13.1), in females 14.5 (13.3-15.6). The mean dose was 
higher in 1999-2001 than 1995-1998. Serum sodium, potassium, calcium, magnesium, phosphate, and bicarbonate concentrations were well-maintained. $55 \pm 29$ bags were discarded per month; $7 \%$ from prescription change, $62 \%$ expiration at 72 hours, $6 \%$ stopped CRRT, $24 \%$ bag leak. No random dialysate monitoring test was positive. No dialysate related complications or reactions were reported. Total cost per liter (materials + labor) in 1995 was $\$ 0.91$ and $\$ 0.67$ in 2005. Conclusion: ICU patients with ARF can be supported on CRRT-HD mode with machine generated, and customized, bicarbonate-based dialysate.

\section{0}

Vascular Access in Critically III Patients with Acute Renal Failure Treated with Hemodialysis/Hemofiltration

\section{R. Ponikvar, N. Skofic, J. Buturovic-Ponikvar \\ Department of Nephrology, University Medical Center, University of Ljubljana, Slovenia}

The purpose of this retrospective clinical study was to evaluate complication rate of hemodialysis (HD) catheters which have been inserted between January and October 2005 as acute vascular access in critically ill patients with acute renal failure having been treated with HD. Patients and Methods: In 84 critically ill patients, 59 men, 25 women, aged from 36 to 92 years (mean $68 \pm 11$ ), totally $155 \mathrm{HD}$ catheters have been inserted: 89 single lumen, 55 double lumen into femoral vein; 2 single lumen, 6 double lumen into jugular vein; 1 single lumen, 2 double lumen into subclavian vein. Real time ultrasound guidance was used for the insertion of jugular/subclavian catheters. Mupirocin ointment was applied at the exit site. Single lumen catheters were primed with $4 \%$ and double lumen with $30 \%$ trisodium citrate. Results: Catheters were inserted for totally 1722 days and $1137 \mathrm{HD}$ have been performed. Insertion was free of complications. In $36 / 155(23.2 \%)$ catheters the following side effects were observed: $30(19.4 \%)$ catheters had to be removed and replaced over a guidewire (thrombosis of the lumen in 17 catheters $/ 11.1 \% /$, thrombosis of the vein in 1 catheter $/ 0.6 \% /$, mechanical damage in 5 catheters/ $3.2 \% /$, avulsion in 3 catheters $/ 1.9 \% /$, suspected exit site infection in 2 catheters $/ 1.3 \% /$ and suspected catheter related septicemia in 2 catheters $/ 1.3 \% /)$, in $3(1.9 \%)$ r-TPA was applied for thrombolysis, and in $6(3.9 \%)$ flushing with saline and transient change in blood flow direction reversed malfunction (low blood flow). Overall complication rate was 20.9/1000 catheter days. Complication rate for single lumen catheters $(10.7 / 1000$ catheter days $)$ was significantly lower vs. double lumen $(24.4 / 1000$ catheter days $)(p<0.05)$. Complication rate for single lumen femoral catheters (11/1000 catheter days) was significantly lower vs. double lumen femoral catheters $(24.4 / 1000$ catheter days, $\mathrm{p}<0.05)$. Overall complication rate of femoral catheters compared to subclavian and jugular catheters was not significantly different $(15.6 / 1000$ vs. $16.8 / 1000$ catheter days, $p=0.92)$. There were 1.16 exit site infections/1000 catheter days and no catheter related septicemia. Conclusions: Significantly higher complication rate was observed in double lumen catheters. No difference was observed in complication rate between femoral and subclavian/jugular catheters.

\section{1 \\ CRRT in Leptospirosis in Developing Countries}

\begin{abstract}
M. Leelakumari, V. Avadaiammal, J. George,
B. Upendran, M.K. Mohandas, S. Varma
\end{abstract}

Medical College Hospital, Trivandrum, India

Leptospirosis is an important cause of acute renal dysfunction in southern parts of Kerala which is a state in South India. Leptospirosis causes multiorgan dysfunction with involvement of kidney, liver, heart and blood components with hemodynamic instability being very common. In our study, we attempted to define the role of CRRT in altering the outcome in patients with leptospirosis and hemodynamic instability. All patients with clinically suspected leptospirosis admitted to Medical College Hospital, Trivandrum from January 1, 2003 to December 31, 2004 were taken for the study. Patients who underwent CRRT for less than 6 hours were excluded. There were 22 patients in the study group with a mean age of $46.9 \pm 15.4$ years. Male to female ratio was $3.4: 1$. All patients had multiple organ involvement. 12 patients $(54 \%)$ required more than one sitting of CRRT. CVVHDF was the most common modality of CRRT employed. $63.6 \%$ patients survived. Follow-up of these patients at 6 weeks showed normal renal, liver, cardiac and hematological parameters. We conclude that CRRT remains an important modality of renal support in patients with leptospirosis with renal involvement and multiorgan dysfunction in developing countries. The limitations to the administration of CRRT were the patient's financial constraints which necessitated the use of PD fluid (which was prepared inside the hospital) as dialysate fluid, the use of F8 filter instead of CVVH filter and the lack of facilities for continuous monitoring of biochemical parameters and blood gases. We also used an ordinary blood pump instead of sophisticated CRRT equipment available in developed countries.

32

\section{Implementation of $35 \mathrm{ml} / \mathbf{k g} / \mathrm{h}$ of CRRT Dose in ICU: A Combined Medical and Nursing Approach}

\author{
P.M. Honoré ${ }^{1}$, O. Joannes-Boyau², T. Kotulak ${ }^{3}$, W. Boer 4 , \\ D. Renard ${ }^{1}$, C. Verly ${ }^{1}$ \\ ${ }^{1} \mathrm{ICU}$, St-Pierre-Para-Universitary Hospital, Ottignies-LLN, \\ Belgium; ${ }^{2} \mathrm{ICU}, \mathrm{DAR}$ II, University of Bordeaux, France; \\ ${ }^{3} \mathrm{ICU}$, Charles University, Prague, Czeck Republic, \\ ${ }^{4} \mathrm{CU}$, Atrium Hospital, Heerlen, Netherlands
}

Objectives: Describe all the rigorous education process performed inside the nursing and the medical team in order to make possible this change from $20 \mathrm{ml} / \mathrm{kg} / \mathrm{h}$ up to $35 \mathrm{ml} / \mathrm{kg} / \mathrm{h}$. Background: In early 2002 , we decide to implement $35 \mathrm{ml} / \mathrm{kg} / \mathrm{h}$ as a standard dose of CRRT in all our patients according to the study of Ronco publish in the lancet 2001. For the past 15 years, we have been using CRRT with BSM 22, BM 25, Prisma and finally Aquarius. A nursing cell specially dedicated to CRRT has been created in our unit more than ten years ago. This cell allow us to have dedicated nurses to CRRT in every shift. This is very helpful also when we do need to implement new machines or 
new techniques. Such a change need a rigorous education process in the nursing cell first and than to the whole nursing team as well physicians including colleagues. Setting: General Hospital, Regional Centre of 450 beds, Interland population of 150,000 inhabitants and a medicalsurgical general ICU of 15 beds having 1000 admissions a year. The Unit is performing from 40 CRRT patients up to 50 CRRT patients per year. Design: Descriptive study and reported single centre experience. Changes Made in the Daily Practice: (1) Vascular Access: need of a co-axial catheter of 14 french. The catheter site had to be exclusively a Right internal Jugular Approach (Posterior one) with a $20 \mathrm{~cm}$ length and the tip of the catheter placed in the right atrium. Indeed, in order to perform $35 \mathrm{ml} / \mathrm{kg} / \mathrm{h}$ in each patient regardless of the body weight (from $50 \mathrm{~kg}$ up to $150 \mathrm{~kg}$ ), we need a blood flow of $300 \mathrm{ml} / \mathrm{min}$ in order to keep the filtration fraction around and below 30\%. Indeed for a $50 \mathrm{~kg}$ patient $=35 \times 50=1,7501 / \mathrm{h}$ which gives an $\mathrm{FF}$ of less than $15 \%$ but for a $150 \mathrm{~kg}$ patient $=35 \times 150=5,250 \mathrm{l} / \mathrm{h}$, this gives an FF of $29 \%$. Yan can obviously not keep changing your blood flow upon the body weight of the patient. You need to stick to very strict rules. The choice of the RIJ approach need a very large consensus within all the ICU consultants and again very strict rules have to be applied. (2) Preand Post-Dilution Policy: according to the latest literature, we did choose $33 \%$ of pre-dilution and $66 \%$ of post-dilution. This policy allow us to prevent some clotting and some cloogging by the use of a certain amount of predilution. This policy also permits to sustained a good convection rate. Indeed, the loss of convection by the concomitant use of pre-dilutiuon reduces the risk of fouling and protein cake formation and finally preserve further loss of convection. (3) Pre-determination of the Exchange rate according the body weight: in order to reduce the workload at the beginning of the CRRT, we did introduce full tables which can give automatically the amount of pre- and post for a given body weight eliminating the need of sophisticated calculation that can lead to further mistakes. Additional tables do exist also regarding the adaptation of antibiotics to $35 \mathrm{ml} / \mathrm{kg} / \mathrm{h}$ and other drugs as well. (4) No further need of associate dialysis: during the last three years, even in very severe rhabdomyolysis cases, no need of associate dialysis was required whatever the release of potassium was. This allow us to use the aquarius with combined pre-and post-dilution and also to save money regarding additional cost of fluids. Conclusions: This descriptive and reporting study is showing that the implementation of new guidelines as the $35 \mathrm{ml} / \mathrm{kg} / \mathrm{h}$ rule can not be decided in one day. This will need a rigorous lonstanding educational process as well for the nursing staff and for the medical team. A nursing cell can be of help in order to achieved this. We will embark soon upon the implementation of citrate using the same implementation and educational process.

\section{3 \\ Efficacy and Safety of Regional Citrate Anti-coagulation in High-flow CVVH}

\author{
S.R. Sanghvi, B. Becker, A. Brunson, R.M. Hofmann \\ University of Wisconsin, Madison, WI, USA
}

Purpose of the Study: Continuous Renal Replacement Therapy (CRRT) improves critically ill patient's survival when ultra-filtration (UF) exceeds $35 \mathrm{ml} / \mathrm{kg} / \mathrm{h}$. This study evaluated the safety and efficacy of citrate regional anti-coagulation in high dose continuous veno-venous hemofiltration (CVVH). Methods Used: We initiated a new high flow CVVH protocol utilizing citrate for regional anti-coagulation in September 2004. After our new protocol had been used for a year, we randomly chose two consecutive months to retrospectively evaluate the efficacy and safety of our newly implemented citrate anti-coagulation protocol. Patients were placed on the NxStage system one (NxStage Medical, Lawrence, MA) with a blood flow (Qb) of $250 \mathrm{ml} / \mathrm{min}$ and replacement fluid (Qrf) rate of 3 liters/hr. The primary renal team adjusted $\mathrm{Qb}$ and Qrf as clinically deemed appropriate. The primary endpoint was filter life. Secondary endpoints included UF rate, electrolyte abnormalities, and citrate toxicity. Laboratory values were followed every 8 hours while on citrate anti-coagulation including sodium, bicarbonate, BUN, creatinine, total calcium as well as serum and post filter ionized calcium levels. Summary: 56 systems (filters) utilizing citrate anti-coagulation and 12 systems (filters) utilizing standard heparin anticoagulation in a total of 19 patients were evaluated. Patients developed renal failure from multiple causes. The citrate systems and heparin systems average filter lives were 33 hours and 24 hours respectively $(\mathrm{p}=0.09$ ). After censoring for interruption due to procedures, catheter failures, expiration of the patient or system no longer needed, there were a total of 39 systems with citrate anti-coagulation and 9 systems with heparin anticoagulation. The average filter life of the citrate vs. heparin system was 40 hours vs. 31 hours $(\mathrm{p}=0.09)$ respectively. The average UF rate was $49 \mathrm{ml} / \mathrm{kg} / \mathrm{min}$. There were no cases of citrate toxicity. Review of electrolyte safety revealed one incidence of metabolic alkalosis, one incidence of hyponatremia, one incidence of hypokalemia, one incidence of hyperkalemia. No incidences of hypernatremia occurred. Conclusions: Citrate anti-coagulation for high-flow CVVH is safe and efficacious. The filter life is prolonged vs. heparin anticoagulation. Electrolyte abnormalities are minimal with frequent surveillance. There were no cases of citrate toxicity, despite several patients with advanced cirrhosis.

\section{4}

\section{Citrate Anticoagulation for Fresenius CRRT}

\author{
J. Schreiner ${ }^{1}$, R. Frank' 1 D. Duffy ${ }^{2}$ \\ ${ }^{1}$ St Joseph's Hospital, Marshfield, ${ }^{2}$ Marshfield Clinic, \\ Marshfield, WI, USA
}

Purpose of Study: Regional extracorporeal anticoagulation with continuous citrate and calcium chloride can be performed easily, safely and is efficacious. We describe our protocol for this technique and summarize our results for the previous year. Methods: Demonstrate our program and discuss implementation results. Summary: CRRT with this system has been done at our institution since April 13, 2002. A dialysis RN does the initial setup, a daily assessment and is the support person for the ICU/CC (intensive care unit/critical care) RN doing the CRRT treatment, along with the supervising nephrologist. Citrate is run continuously into a Y connect at the catheter hub, into the CRRT system and calcium chloride into a $\mathrm{Y}$ connect at the venous catheter hub, or through a separate central line if systemic anticoagulation is prescribed. A zero calcium dialysate is used. Monitoring with ionized calcium levels and a sliding schedule for the calcium chloride drip is used by the $\mathrm{RN}$. Ease of use, problem solving, calls, and safety issues will be discussed. CRRT was performed on 71 patients between October 2004 through September 2005 for 617 CRRT $24 \mathrm{hr}$ treatments. Conclusion: Citrate anticoagulation with the Fresenius CRRT system is efficacious, relatively simple and safe to use. 
Table for Abstract 35

\begin{tabular}{clllllll}
\hline \# Pts & Days on CRRT & Tech prob & Bleeding prob & Cath changed & Yeast in drain & SUN mg/dl & eGFR on CRRT \\
\hline 30 & $<5$ & No & No & No & Rarely & $<20$ & $30-70$ \\
31 & $5-10$ & No & No & No & Periodic & $<20$ & $30-90$ \\
9 & $10-20$ & No & No & Yes & Periodic & $<20$ & $>90$ \\
2 & $20-30$ & No & No & Yes & Periodic & $<20$ & $>90$ \\
1 & $30-40$ & No & No & Yes & Periodic & $<20$ & $>90$ \\
1 & $50-60$ & No & No & Yes & Periodic & $<90$ \\
\hline
\end{tabular}

35

\section{Fresenius CRRT for Extended Critical Care} Treatment Support

\author{
J. Schreiner ${ }^{1}$, R. Frank ${ }^{1}$, D. Duffy ${ }^{2}$ \\ ${ }^{1}$ St Joseph's Hospital, Marshfield, ${ }^{2}$ Marshfield Clinic, \\ Marshfield, WI, USA
}

Purpose of Study: CRRT for the unstable patient in critical care. We describe our protocol for this technique and summarize our results for the previous year. Methods: Demonstrate our program and discuss implementation results. Summary: CRRT with this system has been done at our institution since April 13, 2002. A dialysis $\mathrm{RN}$ does the initial setup, a daily assessment and is the support person for the ICU/CC (intensive care unit/critical care) RN doing the CRRT treatment, along with the supervising nephrologist. Over the period of 12 months from 10/01/2004 through 9/30/2005 we had 71 patients on CRRT 24/7 for extended treatment each listed as days, and a total of 617 treatments for the year. Conclusion: Extended periods of CRRT can be done to maintain fluid balance and control uremia.

36

Ready to Use vs. Pharmacy Made Dialysate Solution for Continuos Renal Replacement Therapy

\author{
M.B. Shirk, B.S. Martin, D. Smeenk, G. Shidham \\ Ohio State University Medical Center, Columbus, OH, USA
}

Purpose of Study: To evaluate the utility of ready to use dialysate in place of pharmacy made dialysate solutions for CRRT with particular attention to electrolyte balance, electrolyte replacement, workload of pharmacy, nursing workflow, and impact on cost. Method: An evaluation of ready to use dialysate solutions was conducted at the Ohio State University Medical Center. Data was collected on 24 patients for whom ready to use dialysate and electrolyte replacement protocol was used. Data collection included: ready to use formulation (4 formulations available), hours on CRRT, electrolyte levels, and hours above or below target electrolyte range. This data was compared to an historical control group of 20 patients. A survey was distributed to MICU/SICU nurses $(n=41)$ to collect their opinion on the use of ready to use dialysate solutions. An economic evaluation of ready to use and pharmacy made dialysate was completed by a Value Analysis Facilitator. Results: Ready to use dialysate formula

The 11th International Conference on

Continuous Renal Replacement

Therapies (CRRT) with $4 \mathrm{mEq} / \mathrm{L}$ potassium and $2.5 \mathrm{mEq} / \mathrm{L}$ calcium was used for $97 \%$ of total doses (517/535). In the historical control group, 26 different combinations of pharmacy made dialysate solutions were used in 20 patients. Ready to use dialysate plus electrolyte replacement protocol led to a decrease in percentage of time patients spent with potassium $<3.5 \mathrm{mmol} / \mathrm{L}$ as compared to pharmacy made dialysate solution $(10.7 \%$ vs. $18.3 \%)$. There was a reduction in rate of potassium replacement doses ( 0.026 vs. 0.033 replacements/hr). Nursing satisfaction with ready to use dialysate was very high. The nurses survey revealed a perceived improvement in workflow (33/41), in patient care $(31 / 41)$, and a sense that the ready to use dialysate was a positive change (38/41). Cost analysis (material plus labor) showed that pharmacy made dialysate was significantly more costlier than ready to use dialysate solution. Conclusions: Our evaluation of ready to use dialysate plus electrolyte replacement protocol showed to be effective in managing electrolyte balance, reduced the number of electrolyte replacement doses, decreased pharmacy workload, and resulted in a perception of increased nursing time for patient care, thereby serving as potential means to reduce error. In addition, the change offered an anticipated cost savings.
37

\section{A Technique for Rapid Exchange of a Continuous Renal Replacement Therapy}

S. Murphy ${ }^{1}$, M. Ludlow' ${ }^{1}$ A. Chua ${ }^{2}$, S. Alexander ${ }^{3}$, P. Yorgin ${ }^{4}$

'Stanford Medical Center, Stanford, CA, ${ }^{2}$ Texas Children's Hospital, Houston, Texas, 'Lucile Packard Children's Hospital, Stanford, CA, ${ }^{2}$ Loma Linda University Children's Hospital, USA

Re-initiation of continuous renal replacement therapy (CRRT) in neonates and young infants weighing less than 15 kilograms often necessitates a blood prime of the blood circuit path or a concurrent packed red blood cell (PRBC) transfusion to avoid causing hemodynamic instability due to acute hemodilution. The significant amount of time required for a routine CRRT circuit change can be associated with worsening electrolyte and acid-base abnormalities, fluid retention, fever, greater hemodynamic instability and reducing effective hemofiltration time. In an attempt to limit the time without CRRT and to eliminate the requirement for additional blood exposure, a new technique, rapid exchange of CRRT (RECRRT), was developed. Rapid exchange of CRRT is a sequential technique which transfers citrated blood from one CRRT machine to another machine connected in 
series. The technique effectively negates the requirement for CRRT circuit path blood priming or PRBC transfusion. The amount of time without CRRT is markedly reduced by RECRRT to $2-3$ minutes. The RECRRT technique has been utilized more than 30 times for 15 patients without an adverse event. RECRRT may benefit children who weigh less than 15 kilograms and in those patients who experience hemodynamic or clinical instability while CRRT is discontinued for only a brief period.

\section{8}

\section{Calciphylaxis Treated with Sodium Thiosulfate and Continuous Veno-venous Hemofiltration (CVVH)}

\author{
M.L. Bentley $1,2,3$, M. Mangray', J.S. Cain ${ }^{4}$ \\ Carilion Health Systems, Department of ${ }^{1}$ Medical \\ Education and ${ }^{2}$ Pharmacy, Roanoke, Virginia; ${ }^{3}$ Virginia \\ Commonwealth University, School of Pharmacy, \\ Richmond, Virginia, ${ }^{4}$ Valley Nephrology Associates, \\ Roanoke, Virginia, USA
}

Purpose: To report a case of calciphylaxis treated with sodium thiosulfate and CVVH. Material and Methods: A 79-year old woman was admitted after developing hypotension following hemodialysis. Hemodynamic support was required and antibiotics were initiated. An area suggestive of calciphylaxis was present on the abdominal pannus characterized by a large band-like area of painful indurated necrosis without ulceration. Intravenous sodium thiosulfate and intermediate hemodialysis (IHD) was initiated, followed soon thereafter by CVVH with an ultrafiltration rate of $3 \mathrm{~L} / \mathrm{h}$. She was subsequently converted to IHD with sodium thiosulfate. After an additional $3 \mathrm{IHD} /$ sodium thiosulfate treatments, she was discharged. The area of calciphylaxis was painless, less indurated and erythematous, and without ulceration. IHD and sodium thiosulfate were continued. Twenty-four days later she was found to have painful, weeping non-healing ulcers and was readmitted. Surgical consultation was obtained, and she was treated with antibiotics, IHD and sodium thiosulfate. She expired 10 days later after the family elected comfort care. Discussion: Calciphylaxis is a lifethreatening condition that occurs in approximately $1 \%$ of chronic renal failure patients. The mortality rate is approximately $30 \%$ in those without ulceration, but, approaches $80 \%$ once ulceration is present. Although the pathogenesis had not been fully elucidated, it is believed that vascular calcium deposits play a role. As such, treatment has focused on the reduction in levels of serum calcium, phosphorous and their product. Other recommendations have included the use of a low-calcium dialysisate, cessation of calcium-containing phosphate binders and vitamin D supplements, parathyroidectomy, and the use of prednisone if ulceration is not present. Recently, intravenous sodium thiosulfate has shown promise. Thiosulfate is extremely soluble and is believed to allow the dissolution of insoluble calcium salts. Once mobilized, these salts are cleared by CVVH. Conclusion: It is unclear whether combination or individual therapy resulted in initial improvement. However, given that her lesions were non-ulcerative at the time of discharge but severely ulcerative after 3 weeks of IHD with sodium thiosulfate suggest CVVH may play an important role. Future study is needed to elicit whether one modality or its combination is of most benefit.
39

\section{Safety of $\mathbf{0 . 5 \%}$ Isotonic Citrate Replacement Solution for Continuous Venovenous Hemodiafiltration (CVVHDF)}

\author{
V. Vidyasagar' ${ }^{1}$ K.M. Wille', R.R. Speer ${ }^{2}$, K.R. Lai \\ A.J. Tolwani \\ ${ }^{1}$ Carraway Methodist Medical Center, ${ }^{2}$ University of Alabama \\ at Birmingham, Birmingham, AL, USA
}

Purpose: While commercial citrate solutions are approved for blood bank applications, there are currently no FDA-approved citrate solutions for CRRT. Furthermore, available citrate solutions in the U.S. are concentrated and hypernatremic. Use of an isotonic, dilute citrate solution offers two distinct advantages in CRRT: (1) a lower incidence of hypernatremia; and (2) a minimal risk of citrate-related metabolic complications with higher infusion rates. This study reviews the safety of a $0.5 \%$ isotonic citrate solution delivered as replacement fluid with commercially available dialysates in CVVHDF. Methods: A retrospective review of patients undergoing CRRT from May 2004-October 2005 at a tertiary center was performed. Data collected included demographics, clinical parameters, diagnosis, renal failure etiology, chemistries, arterial blood gas, coagulation indices and CRRT parameters. Up to 7 days of data following CRRT initiation were analyzed. Primary markers for citrate toxicity were a total calcium/ionized calcium $(\mathrm{Ca} / \mathrm{iCa})$ ratio $>2.5$ and serum ionized calcium (iCa) $<0.8 \mathrm{mmol} / \mathrm{L}$. The primary marker of hypernatremia was serum sodium $(\mathrm{Na})>150 \mathrm{mmol} / \mathrm{L}$. Secondary markers for citrate toxicity included bicarbonate $\left(\mathrm{HCO}_{3}\right)>30 \mathrm{mmol} / \mathrm{L}$ and $\mathrm{pH}>7.5$. Results: Of 413 patients treated with CRRT using $0.5 \%$ citrate, the first 100 were reviewed. Mean age of this cohort was $57 \pm 17$ years, and $35 \%$ were female. Of the 100 patients, $8 \%$ had known cirrhosis at CRRT initiation. Causes of renal failure included: sepsis $(30 \%)$, surgery $(14 \%)$, hypoperfusion $(11 \%)$, multifactorial (11\%), drug-induced (9\%), ESRD (9\%), cardiogenic $(8 \%)$, hepatorenal (4\%), and other (4\%). At CRRT initiation, mean patient weight was $91 \pm 23 \mathrm{~kg}$, mean dose of dialysis was $33 \pm 7 \mathrm{ml} / \mathrm{kg} / \mathrm{h}$, and mean effluent rate $2877 \pm 738 \mathrm{ml} / \mathrm{h}$. An average of 5 days of data per patient was available for analysis. $90 \%$ of patients had no citrate toxicity as per the two primary markers, and only $3 \%$ (2\%-cirrhosis, $1 \%$-cardiogenic) had citrate toxicity as per abnormal values for both primary markers. Of patients with both abnormal iCa and $\mathrm{Ca} / \mathrm{iCa}$ levels, only 2 had cirrhosis, and neither required discontinuation of citrate therapy. $8 \%$ of patients developed $\mathrm{iCa}<0.8 \mathrm{mmol} / \mathrm{L}$ (mean iCa $0.74 \mathrm{mmol} / \mathrm{L}$ ), and $10 \%$ developed $\mathrm{Ca} / \mathrm{iCa}>2.5$ (mean 2.70 ). $4 \%$ of patients had $\mathrm{Na}>150 \mathrm{mmol} / \mathrm{L}$ (peak Na $153 \mathrm{mmol} / \mathrm{L}$ ). In terms of secondary markers, $22 \%$ had a $\mathrm{pH}>7.5$ (7.51-7.60), while $7 \%$ had $\mathrm{HCO}_{3}>30 \mathrm{mmol} / \mathrm{L}$ (peak $\mathrm{HCO}_{3}$ $33 \mathrm{mmol} / \mathrm{L}$ ). However, just $2 \%$ had both abnormally elevated $\mathrm{pH}$ and $\mathrm{HCO}_{3}$ levels. There were no adverse events directly related to $0.5 \%$ citrate. Conclusion: Nearly all patients who received $0.5 \%$ isotonic citrate for CRRT had favorable biochemical profiles during the study period. Preliminary data from this ongoing study suggest that $0.5 \%$ isotonic citrate is a safe and promising option for use as a standardized citrate solution in CRRT therapy. 


\begin{tabular}{|c|c|c|c|c|c|c|}
\hline & \multicolumn{2}{|l|}{$2 \mathrm{~L} / \mathrm{hr}$} & \multicolumn{2}{|l|}{$3 \mathrm{~L} / \mathrm{hr}$} & \multicolumn{2}{|l|}{$4.5 \mathrm{~L} / \mathrm{hr}$} \\
\hline & Urea & $\mathrm{B} 2 \mathrm{M}$ & Urea & $\mathrm{B} 2 \mathrm{M}$ & Urea & $\mathrm{B} 2 \mathrm{M}$ \\
\hline NxStage CVVHD & $30.9 \pm 2.9$ & $20.5 \pm 8.4$ & $48.0 \pm 2.0$ & $20.6 \pm 2.8$ & $75.2 \pm 2.6$ & $23.7 \pm 6.5$ \\
\hline Prisma/M100 CVVHDF* & $31.7 \pm 0.9$ & $14.5 \pm 1.6$ & $46.6 \pm 1.3$ & $15.2 \pm 2.0$ & $60.6 \pm 2.6$ & $20.5 \pm 4.3$ \\
\hline
\end{tabular}

* For Prisma CVVHDF $2 \mathrm{~L}=1 \mathrm{~L}$ HD, $1 \mathrm{~L} \mathrm{HF} ; 3 \mathrm{~L}=2 \mathrm{~L}$ HD, 1L HF; $4.5 \mathrm{~L}=2.5 \mathrm{~L}$ HD, 2 L HF.

Performing CVVH at $3 \mathrm{~L} / \mathrm{hr}$ with the NxStage machine yielded urea clearances of $45.3 \pm 0.8$ and B2M clearances of $19.2 \pm 4.3 \mathrm{ml} / \mathrm{min}$.

\section{0}

\section{Improved Diffusive Solute Clearances with Novel CRRT Equipment and Dialyzer Design}

\author{
J.K. Leypoldt'1, ${ }^{1}$, P.T. Murray ${ }^{3}$, C.D. Kamerath ${ }^{2}$, J.F. Gilson ${ }^{2}$, \\ G. Friederichs ${ }^{4}$ \\ ${ }^{1}$ VA Salt Lake City Health Care System, ${ }^{2}$ University of Utah, \\ Salt Lake City, UT, ${ }^{3}$ University of Chicago, Chicago, IL, \\ ${ }^{4} \mathrm{NxS}$ tage Medical, Lawrence, MA, USA
}

\begin{abstract}
Purpose: Continuous veno-venous hemodiafiltration (CVVHDF), a combination of diffusive and convective therapies, is a commonly practiced modality that achieves high small solute and middle molecule clearances within the cost and regulatory constraints of fluids for direct infusion. We hypothesized that higher clearances of small solutes and middle molecules could be achieved during purely diffusive therapies when using large surface area dialyzers containing membranes with improved clearance capabilities. This study determined small solute and middle molecule clearances at flow rates consistent with critical care applications using the NxStage System One (NxStage Medical) with its next generation membrane technology (PUREMA, $1.46 \mathrm{~m}^{2}$, Membrana $\mathrm{GmBH}$ ) and compared these findings to CVVHDF as conventionally administered. Methods: Clearances were determined for urea and beta-2-microglobulin (B2M) during purely diffusive (CVVHD) therapy from blood and effluent sampling in an in vitro bovine blood model after the addition of urea and human B2M. Blood flow rates were $250 \mathrm{~mL} / \mathrm{min}$. CVVHD dialysate flow rates were 2,3 , and $4.5 \mathrm{~L} / \mathrm{hr}$. Additional studies were also performed during predilution hemofiltration $(\mathrm{CVVH})$ with a replacement fluid flow of $3 \mathrm{~L} / \mathrm{hr}$. Net ultrafiltration was zero; four tests were performed under each condition using 4 separate dialyzers. Experimentally determined clearances were compared to those published previously (Brunet et al, AJKD 1999) using the Gambro Prisma and M100 cartridge. Results: Determined mean \pm SD clearances (in $\mathrm{mL} / \mathrm{min}$ ) for urea and $\mathrm{B} 2 \mathrm{M}$ were as follows in table 1 . Conclusions: Urea clearances (at $3 \& 4.5 \mathrm{~L} / \mathrm{hr}$ ) and B2M clearances (at 2, 3 \& $4.5 \mathrm{~L} / \mathrm{hr}$ ) are higher with the NxStage system in a purely diffusive (CVVHD) mode than during CVVHDF as conventionally administered. These results are achieved in a simpler format (only one source of fluid) and with lower patient exposure to pharmacy compounding or contamination risk.
\end{abstract}

\section{1 \\ Pediatric Plasma Exchange during Extracorporeal Membrane Oygenation: Electrolyte and Coagualtion Monitoring}

\author{
C.H. Cramer II, D.B. Kershaw, T.A. Mottes, R.S. Nievaard, \\ P.D. Brophy \\ C.S. Mott Children's Hospital University of Michigan, \\ Ann Arbor, MI, USA
}

Purpose: Describe the technical aspects and laboratory results of performing therapeutic plasma exchange (TPE) in a 5 year old $(22 \mathrm{~kg})$ male on extracorporeal membrane oxygenation (ECMO). Methods: This patient was 10 months post cardiac transplant for hypoplastic left heart presented with heart failure. He rapidly deteriorated and was initiated on VA-ECMO for cardiac failure secondary to antibody mediated rejection. TPE was initiated to reduce antibody levels. The patient was maintained on pulse methylprednisolone, cyclosporine and mycophenolate mofetil, with dosing supplementation based on anticipated TPE treatment reductions in these drugs. The PRISMA (Gambro) with TPE 2000 filter (Gambro) was used. Arterial access was connected pre-ECMO bladder via a high flux stop-cock and venous return was to the ECMO bladder. Blood flow rate was $100 \mathrm{~mL} / \mathrm{min}(4.5 \mathrm{~mL} / \mathrm{kg} / \mathrm{min})$. The patient received 6 separate one volume plasma exchanges $(3000 \mathrm{~mL}$ total volume) at 500 to $1000 \mathrm{~mL} / \mathrm{hr}$. Patient's total blood volume was $1540 \mathrm{~mL}(70 \mathrm{cc} / \mathrm{kg})$ and ECMO circuit volume was $2000 \mathrm{~mL}$ (estimated patient plasma volume $1043 \mathrm{~mL})$. Calcium chloride $\left(\mathrm{CaCl}_{2}\right)$ infusion $(20 \mathrm{mg} / \mathrm{mL})$ was initiated at $7 \mathrm{~mL} / \mathrm{hr}$, but increased to $14 \mathrm{~mL} / \mathrm{hr}(0.6 \mathrm{~mL} / \mathrm{kg} / \mathrm{hr})$ during TPE + ECMO. Replacement fluid aliquots were: $250 \mathrm{~mL} 5 \%$ albumin alternating with $250 \mathrm{~mL}$ fresh frozen plasma (FFP) for a total of $1500 \mathrm{~mL} \mathrm{5 \%}$ albumin and $1500 \mathrm{~mL}$ FFP. Summary: PRISMA arterial and venous pressures remained between -179 to $-233 \mathrm{mmHg}$ and 71 to $181 \mathrm{mmHg}$, respectively. The trans-membrane filter pressures ranged from 21 to $39 \mathrm{mmHg}$. $\mathrm{CaCl}_{2}$ drip was initiated for concerns of hypocalcemia from FFP containing citrate and calcium depleted 5\% albumin. Initial ionized calcium was $1.11 \mathrm{mEq} / \mathrm{L}$, but follow-up ionized calcium after 30 minutes of therapy and $250 \mathrm{cc}$ of $5 \%$ albumin replacement was $0.96 \mathrm{mEq} / \mathrm{L}$. This occurred once and after increasing the calcium infusion rate to $14 \mathrm{~mL} / \mathrm{hr}$ the ionized calcium remained normal $(1.10-1.22 \mathrm{mEq} / \mathrm{L})$ for the remaining 6 treatments. Hypokalemia and hypomagnesemia occurred during TPE and required frequent monitoring and replacement. A contributing factor was secondary diuresis from furosemide $(0.1 \mathrm{mg} / \mathrm{kg} / \mathrm{hr})$, but both electrolyte levels decreased during TPE +ECMO. PT/INR, PTT and fibrinogen results remained within clinical parameters. Conclusion: TPE in patients on ECMO is technically feasible and can be 
performed without complications. Our experience supports the need for close follow-up of electrolytes in these patients.

Table 1. Representative Lab Results of a Single Pre-, Intra- and PostTPE Treatment:

\begin{tabular}{lclc}
\hline Labs & Pre-TPE & Intra-TPE $^{\mathrm{a}}$ & Post-TPE \\
\hline Ionized calcium (mmol/L) & 1.14 & 1.12 & 1.20 \\
Potassium (mEq/L) & 3.8 & $4.6^{\mathrm{b}}$ & 3.2 \\
Magnesium (mg/dL) & 2.1 & 1.8 & 1.7 \\
Sodium (mEq/L) & 144 & - & 145 \\
Phosphorous (mg/dL) & 4.4 & - & 3.6 \\
Creatinine/BUN (mg/dL) & $2.4 / 104$ & - & $2.2 / 92$ \\
Albumin (g/dL) & 3.2 & 3.0 & 3.2 \\
Hematocrit (\%) & 29.3 & - & 35.1 \\
PT/INR (sec) & $12.9 / 1.3$ & $13 / 1.3$ & $14.2 / 1.5$ \\
PTT (sec) & 78.4 & 60.2 & 79.5 \\
Fibrinogen (mg/dL) & 156 & 162 & 133 \\
\hline
\end{tabular}

a $2 \mathrm{hrs} / 1.5 \mathrm{~L}$ exchange.

bost-replacement $1 \mathrm{mEq} / \mathrm{kg} \mathrm{KCl}$.

\section{CRRT Research}

\section{3 \\ Animal Experiments of Pulsatile Blood Flow for Hemofiltration}

B.G. Min', J.C. Lee' ${ }^{2}$ K. Lee ${ }^{2}$

${ }^{1}$ Department of Biomedical Engineering, College of Medicine, ${ }^{2}$ Interdisciplinary Program in Medical and Biological Engineering Major, Graduate School, Seoul National University, Seoul, Korea

Although the daily home hemodialysis treatment has many clinical efficacies over the conventional intermittent hemodialysis, huge water treatment facility has been a main obstacle to home dialysis. Because the hemofiltration treatment employs several simple replacement fluid bags without huge water treatment facility, some researchers said daily hemofiltration treatment could be an alternative for daily home hemodialysis. In this study, the animal tests were performed in dogs to evaluate the high convection effects of the pulsatile blood flow in reducing the required water requirements in hemodiafiltration. The results show that the use of pulsatile blood flow can preserve the efficiency of dialyser or hemofilter, and reduce the total water volumes. Pulsatile blood flow can retard the reduction of total cell volume by decreasing the protein adsorption on the semi-permeable membrane. We are developing the daily home hemofiltration system integrated with pulsatile blood pumping mechanism. Pulsatile blood pumping mechanism is transferred from artificial heart's pump technology which is free from hemolysis and thrombosis problems, and its flow rate is modified suitable to renal replacement therapy. We expect the daily home hemofiltration therapy using pulsatile-flow pump to complement the inconvenience of in-center hemodialysis and to improve the quality of life of patients.

A new clipped single-bag type bicarbonate replacement fluid set has been developed. In the recent past, double-bags with bicarbonate replacement fluid have been used for hemofiltration and hemodiafiltration. However, caregivers sometimes forget the proper mixing procedure (compressing the bag to break the inner seal separating the two solutions or snapping the inner cap of the bag). This may produce imbalance of electrolyte and acid base. In order to resolve this problem, we have developed a single-bag with a clip. The bag consists of two inner chambers that separate the solutions. The use of this bag is extremely simple and mishap free. When the caregiver is ready to administer treatment, the bag is simply unfolded and the clip automatically detaches, releasing the fluids; thereby, the fluid is accurately mixed and ready to administer to the patient. This design eliminates the additional mixing step necessary which is sometimes overlooked with previous bag designs. In this study the clipped single-bag pressure resistance and shock resistance were tested using different clip sizes. An optimal clip size was with an outer diameter of $16 \mathrm{~mm}$ and thickness of $2 \mathrm{~mm}$. This new clipped single-bag type bicarbonate replacement fluid set will effectively establish proper mixture of the bicarbonate automatically.

\section{4}

\section{High Volume Hemofiltration in the Management of Severe Hyperdynamic Septic Shock}

\author{
R. Cornejo, R. Castro, C. Romero, L. Castillo, M. Andresen, \\ G. Hernandez, P.D. Downey \\ Universidad Catolica de Chile, Santiago, Chile
}

Introduction: Early mortality in septic shock is usually due to refractory hypotension and progressive acidosis unresponsive to fluids and vasopressors. High-volume hemofiltration (HVHF) could have a role in this setting. Experimental and clinical studies suggest a beneficial effect of HVHF in severe hypodynamic septic shock. Nevertheless, the most prevalent condition is hyperdynamic $(75 \%$ of cases) in which high cardiac output and low systemic vascular resistance are present. There are no studies specifically addressing the effect of HVHF in severe hyperdynamic septic shock. Our aim was to 
Table 1 for Abstract 44

\begin{tabular}{|c|c|c|c|c|c|c|c|}
\hline & & $\mathrm{T} 0$ & $\mathrm{~T} 4$ & $\mathrm{~T} 8$ & $\mathrm{~T} 12$ & T18 & $\mathrm{T} 24$ \\
\hline \multirow[t]{2}{*}{$\mathrm{NE}(\mathrm{ug} / \mathrm{k} / \mathrm{min}) \mathrm{NE}(\mathrm{ug} / \mathrm{k} / \mathrm{min})$} & $\mathrm{R}$ & $0.72 \pm 0.5$ & $0.43 \pm 0.3 *$ & $0.27 \pm 0.2 *$ & $0.16 \pm 0.2 *$ & $0.09 \pm 0.1$ & $0.05 \pm 0.1$ \\
\hline & NR & $0.78 \pm 0.5$ & $0.63 \pm 0.6$ & $0.56 \pm 0.4$ & $0.4 \pm 0.2$ & $0.42 \pm 0.2$ & $0.35 \pm 0.2$ \\
\hline \multirow[t]{2}{*}{ Lactate $(\mathrm{mEq} / \mathrm{L})$} & $\mathrm{R}$ & $5.7 \pm 3.4$ & $4.3 \pm 2.9$ & $3.61 \pm 2.1^{*}$ & $3.59 \pm 2 *$ & $3.2 \pm 1.9$ & $2.84 \pm 1.7$ \\
\hline & NR & $5.6 \pm 2.7$ & $6.4 \pm 3.7$ & $7.54 \pm 3.4$ & $7.53 \pm 3.2$ & $7.92 \pm 3.6$ & $7.9 \pm 3.8$ \\
\hline \multirow[t]{2}{*}{ MAP (mmHg) } & $\mathrm{R}$ & $71.8 \pm 8.7$ & $84.2 \pm 9^{*}$ & $81.4 \pm 6$ & $79.1 \pm 4.5$ & $78.5 \pm 9$ & $78 \pm 8$ \\
\hline & NR & $72.8 \pm 14$ & $73.2 \pm 5$ & $69.4 \pm 13$ & $69.8 \pm 8$ & $74.1 \pm 10$ & $77 \pm 12$ \\
\hline
\end{tabular}

assess the effect of short-term (12h) HVHF on norepinephrine (NE) requirements and lactate levels in patients with severe hyperdynamic septic shock. We also compared expected vs. observed ICU mortality. Methods: A prospective, interventional, non-randomized study was carried out in surgical and medical intensive care units of an academic tertiary centre. We included twenty consecutive patients with severe septic shock, previously unresponsive to a full evidence basedmanagement algorithm and with increasing NE requirements $(>0.3 \mathrm{ug} / \mathrm{k} / \mathrm{min})$ and lactic acidosis. The intervention was a singlesession of $12 \mathrm{~h}$ HVHF. We measured changes in NE requirements and perfusion parameters every $4 \mathrm{~h}$ during $\mathrm{HVHF}$ and $6 \mathrm{~h}$ thereafter. Results: Mean baseline NE dose was $0.76 \pm 0.5 \mathrm{ug} / \mathrm{kg} / \mathrm{min}$ and it decreased significantly by $33 \%$ at $4 \mathrm{~h}, 43 \%$ at $8 \mathrm{~h}$, and $63 \%$ at $12 \mathrm{~h}$. Fourteen patients decreased NE requirements and 11 of them decreased lactate levels (responders $=\mathrm{R}$ ). Nine patients did not decrease NE requirements (non-responders $=N R$ ) within $12 \mathrm{~h}$. Table 1 shows the differences found between $\mathrm{R}$ and NR compared to baseline $(\mathrm{T} 0 ; *=\mathrm{p}<0.05)$. Severity scores predicted ICU mortality rate in $60 \%$, significantly higher than the observed $40 \%$, being concentrated in NR $(83 \%$ vs. $21 \%, p<0.001)$. Conclusion: This is the first clinical study in patients with severe hyperdynamic septic shock, treated with HVHF according to an evidence-based management algorithm. It confirms that HVHF may significantly improve hemodynamic and perfusion parameters. A positive effect on mortality it is also suggested.

45

\section{'Pulse' High Volume Hemofiltration in Septic} Shock

O. Joannes-Boyau' ${ }^{1}$ L. Merson', P.M. Honoré2,

C. Fleureau ${ }^{1}$, H. Roze ${ }^{1}$, G. Janvier ${ }^{1}$

${ }^{1}$ University Hospital of Bordeaux, Pessac, France;

${ }^{2}$ St-Pierre Para-University Hospital, Ottignies, Belgium

Introduction: The purpose of this study was to evaluate the effect of 'Pulse' High Volume Hemofiltration (PHVHF) on hemodynamics and outcome in patients with septic shock. The primary end point was the mortality at 28 days, secondary end point was hemodynamic and organ failure evolutions and at last the comparison with previous study [1]. Materials and Methods: Prospective study in two centers (France and Belgium) in which 38 patients with septic shock were included. All patients were treated by PHVHF with ultrafiltration rate of $100 \mathrm{ml} / \mathrm{kg} / \mathrm{h}$ for 4 hours and $35 \mathrm{ml} / \mathrm{kg} / \mathrm{h}$ the next 20 hours. Changes in hemodynamic variables and doses of norepinephrine required were measured regularly and the biological parameters were tracked daily and all data were analyzed using ANOVA for repeated measurements. Patients were defined as hemodynamic responders if catecholamine doses required decreased more than $50 \%$ with hemodynamic improvement. Comparison between expected vs. observed mortality was made by a CHI 2 test. Results: The mean SAPS II was $65( \pm 9)$ and mean age was 60 years $( \pm 15)$. The global observed mortality was $50 \%$ vs. an expected mortality of $77 \%(\mathrm{p}<0.05)$. Twenty three patients were hemodynamical responders with a mortality of $17 \%$ and 15 were not and all died. Responders and non-responders characteristics (SAPS II, age, organ failure...) were comparable at the beginning, excepted the weight, because the responders were significantly lower in body weight ( $70 \mathrm{vs} .85 \mathrm{~kg}$ ). All the parameters (hemodynamic and biologic) improved in the responders group while non-responders had more hepatic failure. Discussion: Observed mortality was significantly better than the expected one. Hemodynamic improvement has a good prognostic value in term of survival. The weight is an important parameter, not only for the hemofiltration dose adaptation but perhaps also for the specific inflammatory status in the overweight population which could explain the hemodynamic response difference. In the previous study, all the patient were responders with HVHF $(50 \mathrm{ml} / \mathrm{kg} / \mathrm{h})$ maintained during 96 hours. PHVHF is more practicable but could be perhaps less effective than maintained continuous HVHF.

\section{Reference}

[1] Joannes-Boyau O, et al: Impact of high volume hemofiltration on hemodynamic disturbance and outcome during septic shock. Asaio J 2004; 50(1):102-109.

\section{6 \\ Hydroxypropyl Beta Cyclodextrin Transmembrane Clearance during in vitro Continuous Venovenous Hemofiltration (CVVH)}

\author{
M.C. Grio ${ }^{1}$, M.D. Churchwell ${ }^{2}$, J.S. Seroogy ${ }^{3}$, S. Barriere $^{3}$, \\ B.A. Mueller \\ ${ }^{1}$ University of Michigan, ${ }^{2}$ University of Toledo, \\ ${ }^{3}$ Theravance, Inc, USA
}

Objectives: Cyclodextrins are cyclic oligosaccharides used to increase solubility of lipophilic drugs, improve bioavailability, resist degradation, or enhance safety profile. 2-Hydroxypropyl beta cyclodextrin (HP-CD) is used in several marketed agents including intravenous and oral itraconazole solutions and an investigational antibacterial agent, telavancin. HP-CD is primarily renally eliminated, therefore its use is not 
recommended in renal failure. It has a large molecular weight which suggests that it may not be removed by renal replacement therapy yet its small volume of distribution $(\sim 18 \mathrm{~L})$ suggests that it may be removed by CVVH. Clinicians using drugs containing HP-CD have concerns about HP-CD accumulation, however, there are no published data regarding HP-CD disposition in CVVH. The objective of this study was to determine CVVH HP-CD transmembrane clearance $(\mathrm{Cl})$ with two commonly used hemofilters at conventional ultrafiltration (UF) rates. Methods: HP-CD Cl was assessed in our validated in vitro CVVH model using citrate anticoagulated, bovine blood and a B. Braun Diapact machine. Telavancin coupled with HP-CD was added to blood and experiments were run using new AN69 (M100, Gambro) and polysulfone (F160NR, Fresenius) hemofilters. UF rates were $1,2,3$ and $6 \mathrm{~L} / \mathrm{hr}$ with sufficient blood flow $(\mathrm{Qb})$ to maintain UF rates $(200-350 \mathrm{~mL} / \mathrm{min})$. Blood samples were collected from the pre-filter line and UF samples from the post-filter UF port. Concentrations of HP-CD in plasma and UF samples were assayed using qualified HPLC-fluorescence detection methods. Clearance was calculated using standard pharmacokinetic equations and differences in $\mathrm{Cl}$ between the filter types were compared using a twotailed, unpaired t-test. Results: See table. Conclusion: HP-CD is substantially cleared by CVVH. The HP-CD Cl increases significantly with increasing UF rate but the $\mathrm{Cl}$ did not differ by hemofilter type. HP$\mathrm{CD} \mathrm{Cl}$ was independent of plasma concentration. $\mathrm{CVVH} \mathrm{Cl}$ at higher UF flows approximates the total $\mathrm{Cl}$ reported in patients with normal renal function. CVVH may prevent HP-CD accumulation even in critically ill patients with acute renal failure.

\begin{tabular}{|c|c|c|c|}
\hline $\begin{array}{l}\text { Blood flow rates }(\mathrm{Qb}) \\
(\mathrm{mL} / \mathrm{min})\end{array}$ & $\begin{array}{l}\text { Clearance: } \\
\text { AN69 } \\
\text { Mean } \pm \mathrm{SD} \\
(\mathrm{n}=3) \\
(\mathrm{mL} / \mathrm{min})\end{array}$ & $\begin{array}{l}\text { Clearance: } \\
\text { polysulfone } \\
\text { Mean } \pm \mathrm{SD} \\
(\mathrm{n}=5) \\
(\mathrm{mL} / \mathrm{min})\end{array}$ & p-value \\
\hline $\mathrm{Qb} 200 \mathrm{~mL} / \mathrm{min}$, UF $1 \mathrm{~L} / \mathrm{hr}$ & $13.6 \pm 2.84$ & $12.7 \pm 2.25$ & 0.66 \\
\hline $\mathrm{Qb} 200 \mathrm{~mL} / \mathrm{min}, \mathrm{UF} 2 \mathrm{~L} / \mathrm{hr}$ & $30.9 \pm 3.71$ & $26.9 \pm 5.99$ & 0.28 \\
\hline $\mathrm{Qb} 300 \mathrm{~mL} / \mathrm{min}$, UF $3 \mathrm{~L} / \mathrm{hr}$ & $31.4 \pm 15.7$ & $38.7 \pm 10.18$ & 0.53 \\
\hline $\mathrm{Qb} 350 \mathrm{~mL} / \mathrm{min}$, UF 6 L $/ \mathrm{hr}$ & $103.2 \pm 16.0$ & $93.6 \pm 8.48$ & 0.41 \\
\hline
\end{tabular}

47

Trace Element Clearance in Critically III Patients Treated with Continuous Venovenous Hemodiafiltration (CVVHDF)

\section{D.A. Pasko1, I.F. Btaiche1, J.C. Jain², B.A. Mueller', M. Churchwel/ \\ ${ }^{1}$ University of Michigan College of Pharmacy Department of Clinical Sciences and University of Michigan Health System, ${ }^{2}$ University of Notre Dame, ${ }^{3}$ University of Toledo College of Pharmacy, USA}

Background: Nutrition support is an integral part of patient care in an ICU. Increasingly, critically ill patients with acute renal failure (ARF) are being treated with CVVHDF. Limited information is available on CVVHDF drug removal, far less is known about nutrient clearance during CVVHDF, particularly clearance of trace elements (TE). The aim of this study was to assess TE clearance in patients being treated with CVVHDF. Methods: TE (chromium, copper, manganese, selenium, zinc) clearance was assessed in ten critically-ill adults (6 males, 4 females) treated with CVVHDF. Mean \pm SD patient age was $47.7 \pm 15.4$ years and weight $80.2 \pm 30.7 \mathrm{~kg}$. Eight patients with ARF and two with ESRD were treated with CVVHDF for an average of 23.8 days (range 0.5-180). Nutrition support consisted of enteral feeding $(n=6)$ or parenteral nutrition $(\mathrm{n}=2)$. The CVVHDF circuit used a polysulfone hemodiafilter (F-160NR, Fresenius n =9, HF-1000, Gambro $n=1)$ with a dialysate flow rate of $2.05 \pm 0.16 \mathrm{~L} / \mathrm{hr}$ and ultrafiltration flow rate of $7.65 \pm 3.0 \mathrm{~mL} / \mathrm{min}$. Samples were collected and stored using trace element-free materials. Samples were assayed for TE concentration using inductively coupled plasma mass spectrometry (ICP-MS). TE saturation coefficients were determined by obtaining a pre- and posthemodiafilter blood samples and an effluent/spent dialysate sample. Average daily TE loss via CVVHDF was calculated and compared to typical daily TE supplementation in adult parenteral nutrition (Multitrace ${ }^{\mathrm{TM}}-5$ Concentrate, American Regent Laboratories, Inc.) Results: See table. Conclusion: Daily CVVHDF TE loss is minimal compared to the TE supplemented in daily parenteral nutrition and may result in TE accumulation. Considering tissue TE redistribution during stress and non-renal elimination routes for some TE, the clinical implications of our results and their possible effects on blood and tissue TE concentrations remain unknown. Adjustment of TE supplementation for CVVHDF patients may be necessary.

\begin{tabular}{llllll}
\hline $\begin{array}{l}\text { Trace } \\
\text { element }\end{array}$ & Chromium & Copper & Manganese & Selenium & Zinc \\
\hline $\begin{array}{c}\text { Clearance } \\
\text { mL/min } \\
( \pm \mathrm{SD})\end{array}$ & $5.37 \pm 2.25$ & $0.455 \pm 0.33$ & $1.71 \pm 3.96$ & $1.58 \pm 1.23$ & $4.05 \pm 1.37$ \\
$\begin{array}{c}\text { Mean } \\
\text { daily TE }\end{array}$ & $4.21 \mathrm{mcg}$ & $0.011 \mathrm{mg}$ & $0.0002 \mathrm{mg}$ & $3.66 \mathrm{mcg}$ & $0.068 \mathrm{mg}$ \\
$\begin{array}{c}\text { loss } \\
\text { Multi- } \\
\text { trace }\end{array}$ & $10.0 \mathrm{mcg}-5$ & $1.0 \mathrm{mg}$ & $0.5 \mathrm{mg}$ & $60 \mathrm{mcg}$ & $5.0 \mathrm{mg}$ \\
$\begin{array}{c}\text { concen- } \\
\text { tration }\end{array}$ & & & & & \\
$\begin{array}{c}\text { Supplied } \\
\text { minus }\end{array}$ & $5.79 \mathrm{mcg}$ & $0.989 \mathrm{mg}$ & $0.499 \mathrm{mg}$ & $56.34 \mathrm{mcg}$ & $4.93 \mathrm{mg}$ \\
lost & & & & & \\
\hline
\end{tabular}


Septic-Acute Renal Failure. Background: Despite recent publications of small case-series [1,2], it has always been questioned whether or not additional anticoagulation is needed in case of APC therapy for Septic-Acute Renal Failure. Our study was aimed at bringing some additional pieces of information regarding this controversial and very practical issue. Setting: General Hospital, Regional Centre of 450 beds, Interland population of 150,000 inhabitants and a medical-surgical general ICU of 15 beds having 1000 admissions a year. The unit is performing from 40 CRRT patients up to 50 CRRT patients per year. Design: Prospective observational evaluation study and reported single centre experience. Materials and Methods: Ten consecutives ICU Septic-Acute Renal Failure Patients (they were eligible at the same time for CRRT at $35 \mathrm{ml} / \mathrm{kg} / \mathrm{h}$ and eligible for Xigris) who were eligible for APC were enrolled in this prospective observational study with evaluation of mechanical filter life, need of additional anticoagulation, clotting testing. Results: There was no need of additional anticoagulation on top of APC in any of the 10 consecutive patients. The filter life was about $54 \pm 6$ hours which was greater than our usual filter life in the unit. The APTT was always above $60 \mathrm{sec}$ in all our 10 patients during APC treatment. No significant bleeding did occur during APC treatment in these 10 cases. Also, we looked very carefully to the circuits, the filters and the venous chambers after cessation of the treatment and founded a significantly reduced clotting of these three parts of the extra-corporeal circuit. So, APC will probably also reduces aberrant clotting (Extra Body DIC) in the circuit, the filter and the venous chamber. So, not only the anticoagulant properties of APC will counted on this but also the reduction of 'Extra Body DIC'. Conclusions: During CRRT for Septic-Acute Renal Failure with Patients on APC, there is no need for additional anticoagulation on top of APC. The filter life appears to be well preserved. Mechanisms will include not only the anticoagulant properties of APC but also the reduction of 'Extra Body DIC'.

\section{References}

[1] van Zanten AR, et al: Ned Tijdsch Geneshed 2001;31:1179-1180.

[2] de Pont AC, et al: Intensive Care Med 2003;29:1205-1206. knowledge no information on TE clearance has been published in pediatric patients. The purpose of this study was to assess TE clearance in pediatric patients requiring CVVHDF. Methods: Data was collected prospectively from five pediatric patients receiving CVVHDF for various indications during their ICU admission. The children were $<18$ years old and greater than $5 \mathrm{~kg}$ (dry weight). All five Prisma circuits used during the study were equipped with either the M60, AN-69 or the HF 1000 membranes, and operated in the CVVHDF mode. Blood flow ranged from $50-180 \mathrm{~mL} / \mathrm{min}$, dialysate rates from $19.2-38 \mathrm{~mL} / \mathrm{min} / 1.73 \mathrm{~m}^{2}$, and effluent rates from $12.1-24 \mathrm{~mL} / \mathrm{min} / 1.73 \mathrm{~m}^{2}$. Once consent was obtained, blood (arterial and venous), dialysate, and effluent samples were obtained from the dialysis circuit. The samples were then analyzed for zinc, copper, chromium, selenium, and manganese concentrations using inductively coupled plasma mass spectrometry (ICP-MS). TE saturation coefficients were calculated using pre- and post-hemodiafilter blood concentrations and effluent/spent dialysate concentrations. Average daily loss including total amounts, and per kilogram amounts, were calculated and compared to normal daily TE supplementation in pediatric parenteral nutrition (PTE-4) to determine net TE administration. Results: Five patients ranging from $10.5-53 \mathrm{~kg}$ and 23 months -15 years of age completed the study. Patient demographics were quite different, as were the indication for CRRT. Table-1 describes the percentage TE loss that is contributed to CVVHDF for each patient. Conclusions: (1) All TE were detected in the ultrafiltrate, however all were minimally cleared by CVVHDF. (2) Daily loss for all TE is far less than what is typically supplemented in parenteral solutions. (3) Upward adjustment of TE supplementation for CRRT loss unwarranted, and indeed, reduced TE supplementation regimens may be necessary.

Table 1 .

\begin{tabular}{llllll}
\hline Patient & $\begin{array}{l}\text { Chromium } \\
(\%)\end{array}$ & $\begin{array}{l}\text { Manganese } \\
(\%)\end{array}$ & $\begin{array}{l}\text { Copper } \\
(\%)\end{array}$ & $\begin{array}{l}\text { Zinc } \\
(\%)\end{array}$ & $\begin{array}{l}\text { Selenium } \\
(\%)\end{array}$ \\
\hline 1 & 13.93 & 0.00 & 0.31 & 0.90 & 3.36 \\
2 & 5.16 & 0.04 & 0.19 & 0.41 & 3.74 \\
3 & 0.01 & 0.01 & 0.01 & 0.01 & 0.01 \\
4 & 0.01 & 0.01 & 0.01 & 0.01 & 0.01 \\
5 & 0.01 & 0.00 & 0.00 & 0.00 & 0.02 \\
\hline
\end{tabular}

\section{9 \\ Trace Mineral Clearance in Pediatric Patients Supported by Continuous Venovenous Hemodiafiltration (CVVHDF)}

D.A. Pasko1, M.D. Churchwell2, I.F. Btaiche ${ }^{3}$, J.C. Jain ${ }^{4}$, B.A. Mueller ${ }^{3}$

${ }^{1}$ University of Michigan Health System, Pharmacy

Services, ${ }^{2}$ University of Toledo College of Pharmacy,

${ }^{3}$ University of Michigan College of Pharmacy,

${ }^{4}$ University of Notre Dame, USA

Introduction: Pediatric intensive care patients increasingly being treated with CRRT like CVVHDF for fluid management, electrolyte imbalances, and sepsis. In all of these situations, provision of appropriate nutritional support is essential. A component of this nutrition is the administration of trace elements (TE). Limited information exists regarding TE clearance by any CRRT, and to our

The 11th International Conference on

Blood Purif 2006;24:247-273
50

\section{Thyroid Hormone Transmembrane Clearance During Continuous Veno-venous Dialysis vs. Hemofiltration}

\author{
T.T. Cornell' , K.E. Papez' ${ }^{1}$, W.E. Smoyer ${ }^{1}$, P.D. Brophy', \\ T.A. Mottes ${ }^{1}$, D.A. Pasko ${ }^{2}$ \\ ${ }^{1}$ University of Michigan Health System, Pediatrics and \\ Communicable Diseases, Intensive Care, ${ }^{2}$ University of \\ Michigan Health System, Pharmacy Services, USA
}

Introduction: This is a case report of a 16 -years-old female that presented in cardiac failure $(\mathrm{EF}<3 \%)$ secondary to hyperthyroidism that required veno-arterial extracorporeal membrane oxygenation (ECMO) support and in-line CRRT for renal support. The patient 
Table 1 for Abstract 51

\begin{tabular}{|c|c|c|c|c|c|c|c|}
\hline $\begin{array}{l}\text { Hormone } \\
\text { (values) }\end{array}$ & $\begin{array}{l}\text { Day } 1 \text { values } \\
\text { pre-TPE }\end{array}$ & $\begin{array}{l}\text { Day } 2 \text { values after } \\
\text { one run TPE }\end{array}$ & $\begin{array}{l}\text { Day } 3 \text { values after } \\
2 \text { runs TPE }\end{array}$ & $\begin{array}{l}\text { Day } 1 \text { pre-TPE- } \\
\text { Day } 3 \text { post } \\
2 \text { runs TPE }\end{array}$ & $\begin{array}{l}\% \text { decrease } \\
\text { change day } 1 \\
\text { vs. day } 3\end{array}$ & $\begin{array}{l}\text { Day } 1 \mathrm{TPE} \\
\text { clearance } \\
(\mathrm{mL} / \mathrm{min})\end{array}$ & $\begin{array}{l}\text { Day } 2 \mathrm{TPE} \\
\text { clearance } \\
(\mathrm{mL} / \mathrm{min})\end{array}$ \\
\hline Total T3 (95-170 ng/dL) & 181 & 104 & 75 & 106 & 58.56 & 30.48 & 32.45 \\
\hline Free T3 $(2.8-5.3 \mathrm{pg} / \mathrm{mL})$ & 13.8 & 8.7 & 5.1 & 8.7 & 63.01 & 33.15 & 31.57 \\
\hline Total T4 $(4.7-12.4 \mathrm{mcg} / \mathrm{dL})$ & 14.9 & 6.8 & 5.3 & 9.6 & 64.43 & 39.55 & 32.44 \\
\hline Free T4 $(0.73-1.79 \mathrm{ng} / \mathrm{dL})$ & 4.65 & 4.65 & 2.83 & 3.62 & 56.12 & 31.56 & 30.57 \\
\hline
\end{tabular}

weighed $69 \mathrm{~kg}$ (dry weight) and was $1.78 \mathrm{~m}^{2}$. The indication for CRRT in this patient was anuric renal failure. There are a few literature reports of thyroid hormone clearance by CRRT. Methods: The patient was placed on CVVHD in-line with the ECMO circuit, using an Optiflux $160\left(1.6 \mathrm{~m}^{2}\right)$ hemodiafilter, blood flow of $210 \mathrm{~mL} / \mathrm{min}$, with a dialysate flow of $2000 \mathrm{~mL} / \mathrm{hour} / 1.73 \mathrm{~m}^{2}$. During this time period samples of blood (arterial and venous) and effluent were obtained. After 30 minutes of hemodialysis the CRRT mode was switched to CVVH, the blood flow rate didn't change, and a new solution was hung for a pre-filter replacement fluid. The hemofiltration rate was also $2000 \mathrm{~mL} /$ hour $/ 1.73 \mathrm{~m}^{2}$. The saturation coefficient was calculated using the equation: $\mathrm{Sa}=\mathrm{Ce} /(\mathrm{Ca}+\mathrm{Cv}) / 2$, where $\mathrm{Ce}$ is the concentration in the effluent, $\mathrm{Ca}$ is the serum concentration of the patient pre-filter, and $\mathrm{Cv}$ is the serum concentration post-filter. Clearance was then calculated using the equation: $\mathrm{Cl}=(\mathrm{Qd}+\mathrm{Qe}) \times$ $\mathrm{Sa}$. The sieving coefficient for the CVVH mode was calculated as $\mathrm{Sc}=\mathrm{Ce} / \mathrm{Ca}$, where $\mathrm{Ce}$ is the concentration in the effluent, $\mathrm{Ca}$ already defined is the serum concentration of the patient pre-filter, prereplacement fluid. Clearance was then calculated using the equation: $\mathrm{Cl}=\mathrm{Sc} \times$ ultrafiltrate rate (UFR). Results: The results are summarized in table 1. Conclusions: Thyroid hormones, free/bound T3/T4 are minimally removed by CVVHD and CVVH. CRRT did remove more free T3 compared to the other thyroid hormones; however this may or may not be clinically significant. CRRT should not be used for the indication of removing thyroid hormones for patients with significant hyperthyroidism.

Table 1.

\begin{tabular}{lllll}
\hline $\begin{array}{l}\text { Hormones } \\
\text { (normals) }\end{array}$ & $\begin{array}{l}\text { Sa } \\
\text { CVVHD }\end{array}$ & $\begin{array}{l}\text { Clearance } \\
\text { CVVHD } \\
(\mathrm{mL} / \mathrm{min})\end{array}$ & $\begin{array}{l}\text { Sc } \\
\text { CVVH }\end{array}$ & $\begin{array}{l}\text { Clearance } \\
\text { CVVH } \\
(\mathrm{mL} / \mathrm{min})\end{array}$ \\
\hline $\begin{array}{c}\text { Total T3 } \\
(95-170 \mathrm{ng} / \mathrm{dL})\end{array}$ & 0.16 & 5.26 & 0.17 & 5.67 \\
$\begin{array}{c}\text { Free T3 } \\
(2.8-5.3 \mathrm{pg} / \mathrm{mL})\end{array}$ & 0.76 & 25.26 & 0.71 & 23.68 \\
$\begin{array}{c}\text { Total T4 } \\
(4.7-12.4 \mathrm{mcg} / \mathrm{dL})\end{array}$ & 0.02 & 0.68 & 0.02 & 0.65 \\
$\begin{array}{c}\text { Free T4 } \\
(0.73-1.79 \mathrm{ng} / \mathrm{mL})\end{array}$ & 0.11 & 3.51 & 0.10 & 3.39 \\
\hline
\end{tabular}


52

Fluid Removal Rate and Mortality in

Pediatric CRRT: A Report of the

Prospective Pediatric Continuous Renal

Replacement Therapy (ppCRRT) Registry Group

D.H. Simon, K. Seidel, S.L. Goldstein, A.N. Chua, M.J.G. Somers, M.A. Baum, T.E. Bunchman,

P.D. Brophy, D. Blowey, J.D. Fortenberry, D. Chand,

F.X. Flores, R. Hackbarth, S.R. Alexander, J. Mahan,

K.D. McBryde, J.M. Symons

ppCRRT Registry Group, Houston, Texas, USA

Previous work has demonstrated that pediatric patients undergoing CRRT who have higher levels of fluid overload at initiation of therapy have an increased risk of mortality. No studies to date have examined the relationship between fluid removal rates and mortality in pediatric CRRT patients. We conducted a retrospective cohort study of 188 patients from 13 US centers using a multivariable logistic regression model to test the hypothesis that higher rates of fluid removal from volume overloaded patients are associated with reduced mortality. Volume status was measured for each subject from the time of ICU admission to CRRT initiation; fluid excess was defined as the difference between total fluid intake and output. Subjects were then divided into cohorts based on the percentage of excess fluid removed in the first 72 hours of therapy: those who had 0 to $50 \%$ excess fluid removed $(n=60), 50 \%$ or more excess fluid removed $(n=56)$, and those in whom no fluid was removed $(n=72)$. The model included the following covariates: GFR at CRRT initiation, age, gender, PRISM II score, \% fluid overload at CRRT initiation, and number of vasopressors used. Patients who had $<50 \%$ of their fluid excess removed by 72 hours had an adjusted odds ratio for mortality of 3.2 $(95 \%$ CI $1.2-8.2, p=0.018)$ compared with patients who had $50 \%$ or more of their excess fluid removed. We conclude that less fluid removal in the first 72 hours of therapy is associated with a greater risk of mortality when controlling for severity of illness. Prospective trials are needed to determine the appropriate fluid removal rates in fluid overloaded pediatric CRRT patients. 
Adikey, G.K. 15

Akcan-Arikan, A. 20, 21

Alexander, S.R. 6, 10, 37, 52

Andresen, M. 44

Avadaiammal, V. 3, 31

Bagnis, C. 26

Barnett, R. 27

Barriere, S. 46

Baum, M.A. 6, 10, 52

Becker, B. 33

Bentley, M.L. 38

Berube, M.V. 12

Bhalla, A.K. 11

Biancone, L. 19

Bihorac, A. 7

Blowey, D. 1, 6, 10, 52

Boer, W. 32, 48

Bonventre, J.V. 20

Bouchard, J. 2

Brophy, P.D. 1, 6, 10, 41, 50, 51,52

Brunson, A. 33

Btaiche, I.F. 47, 49

Bukovsky, R. 16

Bukovsky, R.M. 27

Bunchman, T.E. 1, 6, 10, 52

Buturovic-Ponikvar, J. 28, 30

Cain, J.S. 38

Camussi, G. 19

Cantaluppi, V. 19

Carrey, Z. 8

Castillo, L. 44

Castro, R. 44

Chand, D. 6, 10, 52

Chaparala, H. 16

Chemitiganti, R.R.V. 8

Chen, N. 17, 18

Chen, X. 17

Chua, A.N. 6, 37, 52

Chung, K.K. 14

Churchwell, M.D. 46, 49

Churchwell, M. 47

Cornejo, R. 44

Cornell, T.T. 50, 51

Cramer II, C.H., 41
Demirjian, S.G. 5, 29

Difiglia, M. 8

Douny, N. 48

Downey, P.D. 44

Duffy, D. 34,35

Feng, X. 18

Fleureau, C. 45

Flores, F.X. 6, 10, 52

Fortenberry, J.D. 6, 10, 52

Frank, R. 34, 35

Friederichs, G. 40

Gaikwad, J. 11

Ganzaroli, M. 26

George, J. 3, 31

Gilson, J.F. 40

Giorcelli, G. 26

Glueck, J. 42

Goldstein, S.L. 1, 6, 10, 20, 21, 52

Gona, P.K. 8

Grio, M.C. 46

Gubensek, J. 28

Gupta, Ankur 11

Gupta, Anu 11

Gupta, Ashwani 11

Guzman, L. 23

Hackbarth, R. 1, 6, 10, 52

Han, W. 20

Hansen, E. 42

Harish Reddy, G. 16

Hernandez, G. 44

Heywood, J.T. 22

Ho, A. 22

Hofmann, R.M. 33

Holcomb, J.B. 14

Honoré, P.M. 32, 45, 48

Horvath, E.E. 14

Howey, K. 5

Ito, S. 42

Jain, J.C. 47,49

Janvier, G. 45

Jefferson, L.S. 21

Jin, Q. 25

Joannes-Boyau, O. 32, 45, 48
Kamerath, C.D. 40

Kennedy, C. 21

Kershaw, D.B. 41

Khullar, D. 11

Kotulak, T. 32, 48

Krish, P. 8

Lai, K.R. 39

Layon, A.J. 7

Lee, J.C. 43

Lee, K. 43

Leelakumari, M. 3, 31

Leypoldt, J.K. 40

Loftis, L.L. 20, 21

Longobucco, M.J. 27

Lourtie, A.-M. 48

Ludlow, M. 37

Lukae, H. 9

Luo, H.D. 15

Maccariello, E. 4

Madore, F. 2

Mahan, J. 6, 10, 52

Malik, M. 11

Mangray, M. 38

Mann, E.A. 14

Marangella, M. 26

Martin, B.S. 36

Martinez, J.A. 24

Mathur, V.S. 22, 23

McBryde, K.D. 6, 10, 52

Merson, L. 45

Min, B.G. 43

Miyamoto, H. 42

Mohandas, M.K. 3, 31

Moldawer, L.L. 7

Motomura, T. 42

Mottes, T.A. 41, 50, 51

Mueller, B.A. 46, 47, 49

Murphy, S. 37

Murray, P.T. 40

Nagrik, A.P. 15

Nievaard, R.S. 41

Nogueira, L. 4

Nosé, Y. 42

Ohnishi, Y. 42

\section{KARGER}

(C) 2006 S. Karger AG, Basel

Fax +41 613061234

E-Mail karger@karger.ch

www.karger.com 
Ojha, S. 11

Okahisa, T. 42

Pacitti, A. 19

Paganini, E.P. 5, 29

Papez, K.E. 50, 51

Pasko, D.A. 47, 49, 50, 51

Pejakov, L.J. 9

Petrillo, R.L. 8

Podda, G. 19

Ponikvar, R. 28, 30

Price, M. 23

Raman, A.S. 15

Rana, D.S. 11

Rao, B.K. 11

Ratkovie, M. 9

Ren, H. 17

Renard, D. 32

Renz, E.M. 14

Rocha, E. 4

Romero, C. 44

Roze, H. 45

Sanghvi, S.R. 33

Sastri, P.S. 11
Schreiner, J. 34, 35

Segoloni, G.P. 19

Seidel, K. 52

Seroogy, J.S. 46

Shen, P. 17

Shi, H. 18

Shidham, G. 36

Shirk, M.B. 36

Simon, D.H. 52

Skofic, N. 28, 30

Smeenk, D. 36

Smoyer, W.E. 50, 51

Soares, M. 4

Somers, M.J.G. 6, 10, 52

Soni, S. 15

Speer, R.R. 39

Stephens, B. 14

Suda, Y. 42

Suh, H. 16

Symons, J.M. 6, 10, 52

Teirstein, P. 23

Teo, B.W. 5, 29

Tolwani, A.J. 39

Upendran, B. 3, 31
Valente, C. 4

Vallée, M. 2

Varma, S. 3, 31

Verdecchia, C. 26

Verly, C. 32

Vidyasagar, V. 39

Visweswar Reddy, P. 13

Vitale, C. 26

Vujoševie, V. 9

Wadhwa, N.K. 16, 27

Washburn, K. 20, 21

Wille, K.M. 39

Wolf, S.E. 13

Wright, E. 29

Xie, J. 18

Xu, Y. 18

Yadin, O. 24

Yorgin, P. 37

Zaritsky, J.J. 24

Zhang, W. 17, 18

Zhu, P. 17 